\title{
NOETHERIANITY UP TO CONJUGATION OF LOCALLY DIAGONAL INVERSE LIMITS
}

\author{
ARTHUR BIK
}

\begin{abstract}
АвsтRACт. We prove that the inverse limit of the sequence dual to a sequence of Lie algebras is Noetherian up to the action of the direct limit of the corresponding sequence of classical algebraic groups when the sequence of groups consists of diagonal embeddings. We also classify all conjugation-stable closed subsets of the space of $\mathbb{N} \times \mathbb{N}$ matrices.
\end{abstract}

Throughout this paper, we work over an infinite field $K$. Consider a sequence of groups

$$
G_{1} \longrightarrow G_{2} \longrightarrow G_{3} \longrightarrow \ldots
$$

together with a sequence of finite-dimensional vector spaces over $K$

$$
V_{1} \longleftarrow V_{2} \longleftarrow V_{3} \longleftarrow \ldots
$$

such that $V_{i}$ is a representation of $G_{i}$ and the map $V_{i+1} \rightarrow V_{i}$ is $G_{i}$-equivariant for all $i \in \mathbb{N}$. Then the direct limit $G$ of the sequence of groups naturally acts on the inverse limit $V$ of the sequence of vector spaces. A subset $X$ of $V$ is Zariski-closed if it is the inverse limit of a sequence of Zariski-closed subsets $X_{i} \subseteq V_{i}$. Now one can ask the following question. Given a descending sequence

$$
V \supseteq X^{(1)} \supseteq X^{(2)} \supseteq X^{(3)} \supseteq \ldots
$$

of Zariski-closed $G$-stable subsets of $V$, is there always a $j \in \mathbb{N}$ such that $X^{(i)}=X^{(j)}$ for all $i \geq j$ ?

If the answers is yes, then the space $V$ is called G-Noetherian. See [HS, DE, Eg] for examples of such spaces. The easiest example of a space $V$ that is not $G$-Noetherian is given by an infinite-dimenional vector space acted on by the trivial group. Recently it was proven $[\mathrm{Dr}]$ that polynomial functors of finite degree are Noetherian. Such functors give rise to $G$-Noetherian spaces $V$ where $G_{i}=\mathrm{GL}_{i}$, the map $G_{i} \rightarrow G_{i+1}$ is given by

$$
A \mapsto\left(\begin{array}{cc}
A & \\
& 1
\end{array}\right)
$$

and where $V_{i}$ is a polynomial representation of $\mathrm{GL}_{i}$. This was then generalised [ES] to algebraic polynomial functors of finite degree. Such functors give sequences $\left(G_{i}\right)_{i \geq 1}$ of classical algebraic groups together with algebraic representations $\left(V_{i}\right)_{i \geq 1}$.

2010 Mathematics Subject Classification. 13E99; 14L30; 17B45.

Key words and phrases. Noetherianity; locally finite Lie algebras; classical groups.

The author was partially supported by the NWO Vici grant entitled Stabilisation in Algebra and Geometry. 
In this paper, we consider sequence of classical algebraic groups that do not arise this way, such as the sequence

$$
\mathrm{SL}_{1} \longleftrightarrow \mathrm{SL}_{2} \longleftrightarrow \mathrm{SL}_{4} \longleftrightarrow \ldots \longleftrightarrow \mathrm{SL}_{2^{i}} \longleftrightarrow \ldots
$$

with maps given by

$$
\begin{aligned}
\mathrm{SL}_{2^{i}} & \hookrightarrow \mathrm{SL}_{2^{i+1}} \\
A & \mapsto\left(\begin{array}{ll}
A & \\
& A
\end{array}\right),
\end{aligned}
$$

where the image of an element $A \in G_{i}$ in $G_{i+1}$ can contain multiple copies of $A$. To such a sequence of groups, there is a corresponding sequence of Lie algebras, which we then dualize to get a sequence going in the opposite direction. We prove that the inverse limit of this sequence is Noetherian up to the action of the direct limit of the sequence of groups.

Notation and conventions. Let $\mathbb{N}$ be the set of positive integers. Denote the dual of a vector space $V$ by $V^{*}$. Let $i, j, k, \ell, m, n \in \mathbb{N}$ be integers. Define $\delta_{i j}$ to be 1 if $i=j$ and 0 if $i \neq j$. Denote the set of $n \times n$ matrices by $\mathrm{gl}_{n}$. When $m \leq n$, we write $\operatorname{pr}_{m}$ for the projection map $\mathfrak{g l}_{n} \rightarrow \mathfrak{g l}_{m}$ of $n \times n$ matrices onto their topleft $m \times m$ submatrix. Denote the inverse limit of the sequence

$$
\mathrm{gl}_{1} \longleftarrow \mathrm{gl}_{2} \longleftarrow \mathrm{gl}_{3} \longleftarrow \longleftarrow \ldots
$$

by $\mathfrak{g l}_{\infty}$, let $I_{\infty} \in \mathfrak{g l}_{\infty}$ be the infinite identity matrix and write $\mathrm{pr}_{n}$ for the projection map $\mathfrak{g l}_{\infty} \rightarrow \mathfrak{g l}_{n}$. Denote the set $\{1, \ldots, n\}$ by $[n]$. Let $P, Q \in \mathfrak{g l}_{n}$ be matrices. For subsets $\mathscr{K}, \mathscr{L} \subseteq[n]$, we write $P_{\mathscr{K}, \mathscr{L}}$ for the submatrix of $P$ with rows $\mathscr{K}$ and columns $\mathscr{L}$. We say that $P$ and $Q$ are similar (and write $P \sim Q$ ) if there is a matrix $A \in \mathrm{GL}_{n}$ such that $P=A Q A^{-1}$. We say that $P$ and $Q$ are congruent if there is a matrix $B \in \mathrm{GL}_{n}$ such that $P=B Q B^{T}$. For matrices $P_{1}, \ldots, P_{k}$ not necessarily of the same size, denote the block-diagonal matrix with blocks $P_{1}, \ldots, P_{k}$ by $\operatorname{Diag}\left(P_{1}, \ldots, P_{k}\right)$.

Acknowledgements. I thank Jan Draisma and Michał Lason for the helpful discussions I had with them. I also thank Jan Draisma for finding and proving Proposition 16 and for proofreading this paper. Finally, I thank the anonymous referee for carefully reading this paper and for their useful comments.

\section{The MAIN RESULtS}

We consider sequences of embeddings

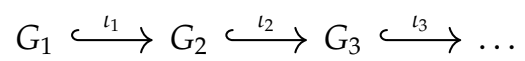

built up out of homomorphisms between the following classical algebraic groups

$$
\begin{aligned}
& \mathrm{A}_{n-1}: \mathrm{SL}_{n} \quad=\left\{A \in \mathrm{GL}_{n} \mid \operatorname{det}(A)=1\right\} \quad \text { for } n \in \mathbb{N}
\end{aligned}
$$

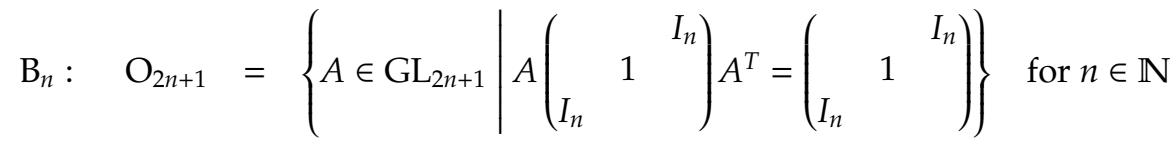

$$
\begin{aligned}
& \mathrm{C}_{n}: \quad \mathrm{Sp}_{2 n}=\left\{A \in \mathrm{GL}_{2 n} \mid A\left(\begin{array}{ll}
-_{n} & I_{n}
\end{array}\right) A^{T}=\left(\begin{array}{cc}
I_{n} \\
-I_{n}
\end{array}\right)\right\} \quad \text { for } n \in \mathbb{N}
\end{aligned}
$$




$$
\mathrm{D}_{n}: \quad \mathrm{O}_{2 n} \quad=\left\{A \in \mathrm{GL}_{2 n} \mid A\left(\begin{array}{cc}
I_{n} \\
I_{n}
\end{array}\right) A^{T}=\left(\begin{array}{cc} 
& I_{n} \\
I_{n} &
\end{array}\right)\right\} \quad \text { for } n \in \mathbb{N}
$$

which we view as embedded subgroups of $\mathrm{GL}_{n}$, for appropriate $n \in \mathbb{N}$. Let $G, H$ be such groups, let $V, W$ be their standard representations and consider $K$ as the trivial representation of $G$. In [BZ], an embedding $G \hookrightarrow H$ is called diagonal if

$$
W \cong V^{\oplus l} \oplus\left(V^{*}\right)^{\oplus r} \oplus K^{\oplus z}
$$

as representations of $G$ for some $l, r, z \in \mathbb{Z}_{\geq 0}$ with $l+r \geq 1$. The triple $(l, r, z)$ is called the signature of the embedding. If $G$ is of type $A$, then the signature of a diagonal embedding $G \hookrightarrow H$ is unique. However, if $G$ is of type $\mathrm{B}, \mathrm{C}$ or $\mathrm{D}$, then the representation $V$ is isomorphic to $V^{*}$. In this case, we will always assume that $r=0$, which makes the pair $(l, z)$ unique, and we also denote the signature by $(l, z)$.

Examples 1. Let $G \subseteq \mathrm{GL}_{n}, H, L$ be classical groups of type A, B, C or D.

(a) For each $B \in G^{2} L_{n}$ with $B G=G B$, the automorphism

$$
\begin{aligned}
G & \rightarrow G \\
A & \mapsto B A B^{-1}
\end{aligned}
$$

is diagonal with signature $(1,0,0)$.

(b) For all matrices $A \in G$, we have $A^{-T} \in G$. The automorphism

$$
\begin{aligned}
G & \rightarrow G \\
A & \mapsto A^{-T}
\end{aligned}
$$

is diagonal with signature $(0,1,0)$.

(c) The composition of any two diagional embeddings $G \hookrightarrow H$ and $H \hookrightarrow L$ is a diagonal embedding $G \hookrightarrow L$.

We will assume the sequence

$$
G_{1} \stackrel{\iota_{1}}{\longleftrightarrow} G_{2} \stackrel{\iota_{2}}{\longleftrightarrow} G_{3} \stackrel{\iota_{3}}{\longleftrightarrow \ldots}
$$

consists of diagonal embeddings. Let $G$ be its direct limit and consider the associated sequence

$$
\mathfrak{g}_{1} \longleftrightarrow \mathfrak{g}_{2} \longleftrightarrow \mathfrak{g}_{3} \longleftrightarrow \ldots
$$

where $\mathrm{g}_{i}$ is the Lie algebra of $G_{i}$. Now, we let $V$ be the inverse limit of the sequence

$$
\mathrm{g}_{1}^{*} \longleftarrow \mathrm{g}_{2}^{*} \longleftarrow \mathrm{g}_{3}^{*} \longleftarrow-\ldots
$$

obtained by dualizing the previous sequence. Then $V$ has a natural action of $G$. If we modify our sequence by replacing

$$
G_{i} \stackrel{\iota_{i}}{\longleftrightarrow} G_{i+1} \stackrel{\iota^{i+1}}{\longleftrightarrow} G_{i+2}
$$

by

$$
G_{i} \stackrel{\iota_{i+1} o_{i}}{\longrightarrow} G_{i+2}
$$

then both the direct limit $G$ and the inverse limit $V$ do not change. So we may replace our sequence of groups by any of its infinite subsequences. Conversely, we can also replace our sequence by any supersequence. Note that there always 
exists an infinite subsquence such that every group in the subsequence is of the same type.

Main Theorem. Assume that one of the following conditions hold:

(a) The group $G_{i}$ has type A for infinitely many $i \in \mathbb{N}$.

(b) The characteristic of $K$ does not equal 2.

Then the space $V$ is G-Noetherian, i.e. for every descending sequence

$$
V \supseteq X_{1} \supseteq X_{2} \supseteq X_{3} \supseteq \ldots
$$

of $G$-stable closed subsets of $V$ there is an $i \in \mathbb{N}$ such that $X_{i}=X_{j}$ for all $j \geq i$.

Remark 2. When we prove the Main Theorem, we may assume that all $G_{i}$ have the same type. When this type is $\mathrm{B}, \mathrm{C}$ or $\mathrm{D}$, we assume that $\operatorname{char}(K) \neq 2$. This way we know that the set of (skew-)symmetric $n \times n$ matrices congruent to some given (skew-)symmetric matrix $A$ equals the set of all (skew-)symmetric matrices whose rank is equal to the rank of $A$. See the proofs of Lemmas 41 and 46 and Proposition 51.

When all $G_{i}$ are of type A and $(l, r)=(1,0)$ for all but finitely many embeddings, the group $G$ equals $S_{\infty}$ and the space $V$ can be identified with a quotient of the set $\mathfrak{g l}_{\infty}$ of $\mathbb{N} \times \mathbb{N}$ matrices. We prove this case of the Main Theorem by classifying all $\mathrm{SL}_{\infty}$-stable closed subsets of $\mathfrak{g l}_{\infty}$.

Definition 3. Define the rank of a matrix $P \in \mathfrak{g l}_{\infty}$ as

$$
\operatorname{rk}(P)=\sup \left\{\operatorname{rk}\left(\operatorname{pr}_{n}(P)\right) \mid n \in \mathbb{N}\right\} \in \mathbb{Z}_{\geq 0} \cup\{\infty\} .
$$

We use the following definition from [DE].

Definition 4. Let $n \in \mathbb{N} \cup\{\infty\}$ and let $Q_{1}, \ldots, Q_{k}$ be elements of $\mathfrak{g l}_{n}$. Define

$$
\operatorname{rk}\left(Q_{1}, \ldots, Q_{k}\right)=\inf \left\{\operatorname{rk}\left(\mu_{1} Q_{1}+\cdots+\mu_{k} Q_{k}\right) \mid\left(\mu_{1}: \cdots: \mu_{k}\right) \in \mathbb{P}^{k-1}\right\} \in \mathbb{Z}_{\geq 0} \cup\{\infty\}
$$

to be the rank of the tuple $\left(Q_{1}, \ldots, Q_{k}\right)$.

Theorem 5. The space $\mathrm{gl}_{\infty}$ is $\mathrm{SL}_{\infty}$-Noetherian. Any $\mathrm{SL}_{\infty}$-stable closed subset of $\mathrm{gl}_{\infty}$ is a finite union of irreducible $\mathrm{SL}_{\infty}$-stable closed subsets and the irreducible $\mathrm{SL}_{\infty}$-stable closed subsets of $\mathfrak{g l}_{\infty}$ are $\mathrm{gl}_{\infty}$ itself together with the subsets

$$
\left\{P \in \mathrm{gl}_{\infty} \mid \operatorname{rk}\left(P, I_{\infty}\right) \leq k\right\},\left\{P \in \mathfrak{g l}_{\infty} \mid \operatorname{rk}\left(P-\lambda I_{\infty}\right) \leq k\right\}
$$

for $\lambda \in K$ and $k \in \mathbb{Z}_{\geq 0}$.

Remark 6. We would like to point out that the $\mathrm{SL}_{\infty}$-Noetherianity of $\mathrm{gl}_{\infty}$ also follows from [ES, Theorem 1.2].

Remark 7. Let $P \in \mathfrak{g l}_{\infty}$ be an $\mathbb{N} \times \mathbb{N}$ matrix such that $\operatorname{rk}\left(P, I_{\infty}\right)<\infty$. Then we have $\operatorname{rk}\left(P-\lambda I_{\infty}\right)<\infty$ for some $\lambda \in K$. If this holds for distinct $\lambda, \lambda^{\prime} \in K$, then

$\infty=\operatorname{rk}\left(\left(\lambda-\lambda^{\prime}\right) I_{\infty}\right)=\operatorname{rk}\left(\left(P-\lambda^{\prime} I_{\infty}\right)-\left(P-\lambda I_{\infty}\right)\right) \leq \operatorname{rk}\left(P-\lambda^{\prime} I_{\infty}\right)+\operatorname{rk}\left(P-\lambda I_{\infty}\right)<\infty$

and hence the $\lambda \in K$ such that $\operatorname{rk}\left(P-\lambda I_{\infty}\right)<\infty$ must be unique. This is the infinite analogue of the statement that an $n \times n$ matrix can have at most one eigenvalue with geometric multiplicity more than $n / 2$.

Remark 8. When we call each of the closed subsets $X \subseteq \mathfrak{g l}_{\infty}$ listed in the theorem irreducible, we mean this in the following sense: if we have

$$
X=Y \cup Z
$$

for (not necessarily $\mathrm{SL}_{\infty}$-stable) closed subsets $Y, Z \subseteq X$, then $X=Y$ or $X=Z$. 


\section{Structure of the PROOF}

In this section, we reduce the Main Theorem to a number of cases and we outline the structure that the proofs of each of those cases share.

2.1. Reduction to standard diagonal embeddings. When the vector space $V$ is finite-dimesional over $K$, the Main Theorem becomes trivial. So we will only consider the cases where $V$ is infinite-dimensional. For all $i \in \mathbb{N}$, let $\left(l_{i}, r_{i}, z_{i}\right)$ be the signature of the embedding $\iota_{i}: G_{i} \hookrightarrow G_{i+1}$. When $G_{i}$ is of type B, C or D, we will assume that $r_{i}=0$. The following lemma tells us that we can assume that $l_{i} \geq r_{i}$ for all $i \in \mathbb{N}$.

Lemma 9. For all $i \in \mathbb{N}$, let $\sigma_{i}: G_{i} \rightarrow G_{i}$ be the automorphism sending $A \mapsto A^{-T}$ and take $k_{i} \in \mathbb{Z} / 2 \mathbb{Z}$. Then the bottom row of the commutative diagram

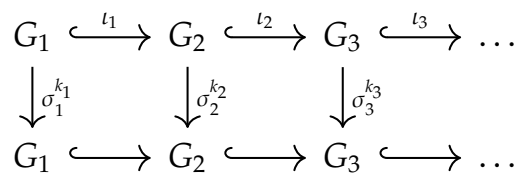

is a sequence of diagonal embeddings with signatures $\sigma^{k_{i}+k_{i+1}}\left(l_{i}, r_{i}, z_{i}\right)$ where $\sigma$ acts by permuting the first two entries.

The lemma follows from the fact that the automorphism $G_{i} \rightarrow G_{i}, A \mapsto A^{-T}$ is diagonal and its own inverse. We can choose the $k_{i}$ recursively so that $l_{i} \geq r_{i}$ for all $i \in \mathbb{N}$ in the bottom sequence. Since the vertical maps are isomorphisms and the diagram commutes, the bottom sequence gives rise to isomorphic $G$ and $V$. This allows us to indeed assume that $l_{i} \geq r_{i}$.

Let $G$ be a classical group of type A, B, C or D. Let $l, r, z \in \mathbb{Z}_{\geq 0}$ be integers with $r=0$ if $G$ is not of type A. Let $\beta_{1}, \beta_{2}$ be non-degenerate $G$-invariant bilinear forms on $V^{\oplus l} \oplus\left(V^{*}\right)^{\oplus r} \oplus K^{\oplus z}$.

Lemma 10. Assume that $K=\bar{K}$ and that one of the following conditions hold:

(a) $\beta_{1}$ and $\beta_{2}$ are both skew-symmetric.

(b) $\beta_{1}$ and $\beta_{2}$ are both symmetric and $\operatorname{char}(K) \neq 2$.

Then there exists a G-equivariant automorphism $\varphi$ of $V^{\oplus l} \oplus\left(V^{*}\right)^{\oplus r} \oplus K^{\oplus z}$ such that

$$
\beta_{2}(\varphi(v), \varphi(w))=\beta_{1}(v, w)
$$

for all $v, w \in V^{\oplus l} \oplus\left(V^{*}\right)^{\oplus r} \oplus K^{\oplus z}$.

Proof. First suppose that $l=r=0$. In this case, the lemma reduces to the wellknown statement that the matrices corresponding to $\beta_{1}$ and $\beta_{2}$ are congruent. In genenal, Schur's Lemma splits the lemma into the cases $r=z=0, l=z=0$ and $l=r=0$ and reduces the first two cases to the third.

Let $f, g: G \rightarrow H \subseteq \mathrm{GL}_{n}$ be two diagonal embeddings with signature $(l, r, z)$.

Lemma 11. If the type of $H$ is $\mathrm{B}, \mathrm{C}$ or $\mathrm{D}$, assume that $K=\bar{K}$. If the type of $H$ is $\mathrm{B}$ or $\mathrm{D}$, assume in addition that $\operatorname{char}(K) \neq 2$. Then there is a $P \in H$ such that the isomorphism 
$\pi: H \rightarrow H, A \mapsto P A P^{-1}$ makes the diagram

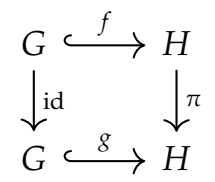

commute.

Proof. The maps $f$ and $g$ both induce an isomorphism

$$
K^{n} \cong V^{\oplus l} \oplus\left(V^{*}\right)^{\oplus r} \oplus K^{\oplus z}
$$

of representations of $G$. This means that there are matrices $Q, R$ such that

$$
Q f(A) Q^{-1}=R g(A) R^{-1}=\operatorname{Diag}\left(A, \ldots, A, A^{-T}, \ldots, A^{-T}, I_{z}\right)
$$

for all $A \in G$ where the block-diagonal matrix has $l$ blocks $A$ and $r$ blocks $A^{-T}$. If $H$ is of type $\mathrm{A}$, then we take $P=\lambda R^{-1} Q$ for some $\lambda \in K$ such that $P \in \mathrm{SL}_{n}$ and see that the isomorphism $\pi: H \rightarrow H, A \mapsto P A P^{-1}$ makes the diagram commute.

Assume that $H$ is not of type A. Then $H=\left\{g \in \mathrm{GL}_{n} \mid g^{T} B g=B\right\}$ for some matrix $B \in \mathrm{GL}_{n}$. Let $\beta_{1}$ and $\beta_{2}$ be the $G$-invariant bilinear forms on $K^{n}$ defined by $Q^{-T} B Q^{-1}$ and $R^{-T} B R^{-1}$. By the previous lemma, there exists a $G$-equivariant automorphism $\varphi$ of $K^{n}$ such that

$$
\beta_{2}(\varphi(v), \varphi(w))=\beta_{1}(v, w)
$$

for all $v, w \in K^{n}$. Let $S$ be the matrix corresponding to $\varphi$. Then

$$
S^{T} Q^{-T} B Q^{-1} S=R^{-T} B R^{-1}
$$

and

$$
S \operatorname{Diag}\left(A, \ldots, A, A^{-T}, \ldots, A^{-T}, I_{z}\right)=\operatorname{Diag}\left(A, \ldots, A, A^{-T}, \ldots, A^{-T}, I_{z}\right) S
$$

for all $A \in G$. Take $P=R^{-1} S^{-1} Q$. Then $P^{-1} \in H$ and therefore $P \in H$. The isomorphism $\pi: H \rightarrow H, A \mapsto P A P^{-1}$ makes the diagram commute.

Proposition 12. For every $i \in \mathbb{N}$, let $\iota_{i}^{\prime}: G_{i} \hookrightarrow G_{i+1}$ be a diagonal embedding with the same signature $\left(l_{i}, r_{i}, z_{i}\right)$ as $l_{i}$. If the type of $G_{i}$ is $\mathrm{B}, \mathrm{C}$ or $\mathrm{D}$ for any $i \in \mathbb{N}$, assume that $K=\bar{K}$. If the type of $G_{i}$ is $\mathrm{B}$ or $\mathrm{D}$ for any $i \in \mathbb{N}$, assume in addition that $\operatorname{char}(K) \neq 2$. Then there exist isomorphisms $\varphi_{i}: G_{i} \rightarrow G_{i}$ making the diagram

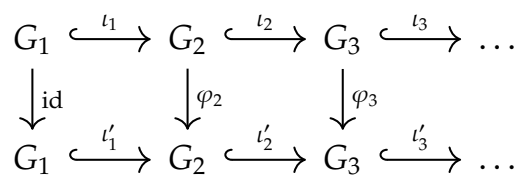

commute.

Proof. We construct the isomorphisms $\varphi_{i}$ recursively in such a way that the $\varphi_{i}$ are also diagonal embeddings with signature $(1,0,0)$. Write $\varphi_{1}=\mathrm{id}$, let $i \geq 2$ and assume that $\varphi_{i-1}$ has has already been constructed. Then $\iota_{i-1}^{\prime} \circ \varphi_{i-1}$ has the same 
signature as $\iota_{i-1}$. So by the previous lemma, there exists an isomorphism $\varphi_{i}$ making the diagram

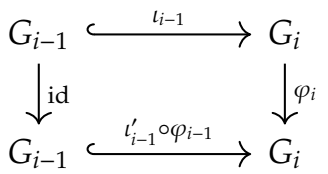

commute that also has signature $(1,0,0)$ as a diagonal embedding.

Recall that, when we replace

$$
G_{1} \stackrel{\iota_{1}}{\longleftrightarrow} G_{2} \stackrel{\iota_{2}}{\longleftrightarrow} G_{3} \stackrel{\iota_{3}}{\longleftrightarrow \ldots}
$$

by supersequences or infinite subsequences, we do not change $G$ or $V$. Therefore we may assume that each group $G_{i}$ has the same type and we will prove the Main Theorem for sequences of groups of type A, B, C and D separately. The proposition tells us that, if we replace $K$ by its algebraic closure, the limits $G$ and $V$ only depend on the signatures of the diagonal embeddings. Since $G$-Noetherianity of $V$ over $\bar{K}$ implies $G$-Noetherianity of $V$ over the original field $K$, we only have to consider one diagonal embedding per possible signature.

2.2. Identifying $V$ with the inverse limit of a sequence of quotients/subspaces of matrix spaces. We encounter the following Lie algebras:

$$
\begin{aligned}
& \mathrm{A}_{n-1}: \mathfrak{s l}_{n} \quad=\left\{P \in \mathfrak{g l}_{n} \mid \operatorname{tr}(P)=0\right\} \quad \text { for } n \in \mathbb{N}
\end{aligned}
$$

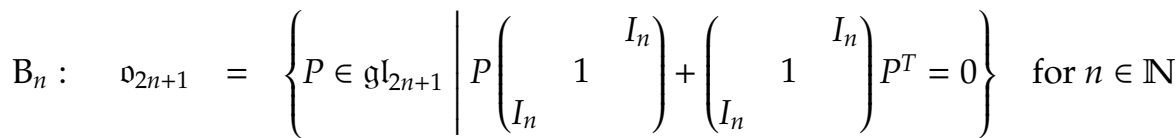

$$
\begin{aligned}
& \mathrm{C}_{n}: \quad \mathfrak{s p}_{2 n}=\left\{P \in \mathfrak{g l}_{2 n} \mid P\left(\begin{array}{ll}
I_{n} \\
-I_{n} &
\end{array}\right)+\left(\begin{array}{rr}
I_{n} \\
-I_{n} &
\end{array}\right) P^{T}=0\right\} \quad \text { for } n \in \mathbb{N} \\
& \mathrm{D}_{n}: \quad \mathfrak{D}_{2 n} \quad=\left\{P \in \mathfrak{g l}_{2 n} \mid P\left(\begin{array}{ll}
I_{n} & I_{n}
\end{array}\right)+\left(\begin{array}{ll}
I_{n} & I_{n}
\end{array}\right) P^{T}=0\right\} \quad \text { for } n \in \mathbb{N}
\end{aligned}
$$

These are all subspaces of $\mathfrak{g l}_{m}$ for some $m \in \mathbb{N}$. Consider the symmetric bilinear form $\mathrm{gl}_{m} \times \mathrm{gl}_{m} \rightarrow K,(P, Q) \mapsto \operatorname{tr}(P Q)$. This map is non-degenerate and therefore the map $\mathfrak{g l}_{m} \rightarrow \mathfrak{g l}_{m}^{*}, P \mapsto(Q \mapsto \operatorname{tr}(P Q))$ is an isomorphism. By composing this map with the restriction map $\mathrm{gl}_{m}^{*} \rightarrow \mathfrak{s l}_{m}^{*}$ and factoring out the kernel, we find that

$$
\begin{aligned}
\mathfrak{g l}_{m} / \operatorname{span}\left(I_{m}\right) & \rightarrow \mathfrak{s l}_{m}^{*} \\
P \bmod I_{m} & \mapsto(Q \mapsto \operatorname{tr}(P Q))
\end{aligned}
$$

is an isomorphism. When $\operatorname{char}(K) \neq 2$ and $\mathfrak{g} \subseteq \mathfrak{g l}_{m}$ is a Lie algebra of type B, C or $\mathrm{D}$, the restriction of the bilinear map to $\mathfrak{g} \times \mathfrak{g}$ is non-degenerate. So the map

$$
\begin{aligned}
\mathfrak{g} & \rightarrow \mathfrak{g}^{*} \\
P & \mapsto(Q \mapsto \operatorname{tr}(P Q))
\end{aligned}
$$

is an isomorphism. Since the map $\mathrm{gl}_{n} \rightarrow \mathfrak{g l}_{n}^{*}$ is in fact $\mathrm{GL}_{n}$-equivariant, the maps $\mathrm{gl}_{m} / \operatorname{span}\left(I_{m}\right) \rightarrow \mathfrak{s l}_{m}^{*}$ and $\mathfrak{g} \rightarrow \mathfrak{g}^{*}$ are all isomorphisms of representations of the groups acting on them. Using these isomorphisms, we identify the duals $\mathfrak{g}_{i}^{*}$ of the 
Lie algebras of the groups $G_{i}$ with quotients/subspaces of spaces of matrices. This in particular allows us to define the coordinate rings of the $\mathfrak{g}_{i}^{*}$ in terms of entries of matrices. For type A, we get

$$
K\left[\mathfrak{g l}_{n} / \operatorname{span}\left(I_{n}\right)\right]=\left\{f \in K\left[\mathfrak{g l}_{n}\right] \mid \forall P \in \mathfrak{g l}_{n} \forall \lambda \in K: f\left(P+\lambda I_{n}\right)=f(P)\right\}
$$

which is the graded subring

$$
K\left[p_{k \ell} \mid k \neq \ell\right] \otimes_{K} K\left[p_{11}-p_{k k} \mid k \neq 1\right]
$$

of $K\left[\mathfrak{g l}_{n}\right]=K\left[p_{k \ell} \mid 1 \leq k, \ell \leq n\right]$. For type $\mathrm{B}$, assuming that $\operatorname{char}(K) \neq 2$, we have

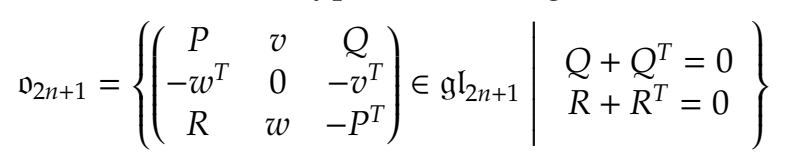

and therefore we get

$$
K\left[\mathfrak{o}_{2 n+1}\right]=K\left[p_{k \ell}, q_{k \ell}, r_{k \ell}, v_{k}, w_{k} \mid 1 \leq k, \ell \leq n\right] /\left(q_{k \ell}+q_{\ell k}, r_{k \ell}+r_{\ell k}\right) .
$$

For type $C$, we have

$$
\mathfrak{s p}_{2 n}=\left\{\begin{array}{lc}
\left(\begin{array}{cc}
P & Q \\
R & -P^{T}
\end{array}\right) \in \mathfrak{g l}_{2 n} & \begin{array}{c}
Q=Q^{T} \\
R=R^{T}
\end{array}
\end{array}\right\}
$$

and we get

$$
K\left[\mathfrak{s p}_{2 n}\right]=K\left[p_{k \ell}, q_{k \ell}, r_{k \ell} \mid 1 \leq k, \ell \leq n\right] /\left(q_{k \ell}-q_{\ell k}, r_{k \ell}-r_{\ell k}\right) .
$$

For type $\mathrm{D}$, assuming that $\operatorname{char}(K) \neq 2$, we have

$$
\mathfrak{D}_{2 n}=\left\{\begin{array}{ll}
\left(\begin{array}{cc}
P & Q \\
R & -P^{T}
\end{array}\right) \in \mathfrak{g l}_{2 n} & \begin{array}{c}
Q+Q^{T}=0 \\
R+R^{T}=0
\end{array}
\end{array}\right\}
$$

and get

$$
K\left[\mathfrak{p}_{2 n}\right]=K\left[p_{k \ell}, q_{k \ell}, r_{k \ell} \mid 1 \leq k, \ell \leq n\right] /\left(q_{k \ell}+q_{\ell k}, r_{k \ell}+r_{\ell k}\right) .
$$

For Lie algebras $\mathfrak{g} \subseteq \mathfrak{g l}_{m}$ of type B, C or D, we will denote elements of $K[\mathfrak{g}]$ by their representatives in $K\left[\mathfrak{g l}_{m}\right]$. Define a grading on each of these coordinate rings by $\operatorname{grad}\left(r_{k \ell}\right)=\operatorname{grad}\left(w_{k}\right)=0, \operatorname{grad}\left(p_{k \ell}\right)=\operatorname{grad}\left(v_{k}\right)=1$ and $\operatorname{grad}\left(q_{k \ell}\right)=2$ for all $k, \ell \in[n]$.

2.3. Moving equations around. Let $X \subsetneq V$ be a $G$-stable closed subset. For each $i \in \mathbb{N}$, let $V_{i}$ be the vector space (we identified with) $\mathfrak{g}_{i}^{*}$ which is acted on by $G_{i}$ by conjugation and let $X_{i}$ be the closure of the projection from $X$ to $V_{i}$. Then $X_{i}$ is a $G_{i}$-stable closed subset of $V_{i}$ for all $i \in \mathbb{N}$ and there exists an $i \in \mathbb{N}$ such that $X_{i} \neq V_{i}$. This means that the ideal $I\left(X_{i}\right) \subseteq K\left[V_{i}\right]$ is non-zero. Let $f$ be a non-zero element of $I\left(X_{i}\right)$ and let $d$ be its degree. The first step of the proof of the Main theorem is to use this polynomial $f$ to get elements $f_{j}$ of $I\left(X_{j}\right)$ such that $f_{j} \neq 0$, such that $\operatorname{deg}\left(f_{j}\right) \leq d$ and such that $f_{j}$ is "off-diagonal" for all $j \gg i$. When the groups $G_{i}$ are of type $\mathrm{B}, \mathrm{C}$ or $\mathrm{D}$, this last condition means that $f_{j}$ is a polynomial in only the variables $r_{k \ell}$ and $w_{k}$. When the groups $G_{i}$ are of type A, we similarly require that the $f_{j}$ are polynomials in the variables $p_{k \ell}$ with $k \in \mathscr{K}$ and $\ell \in \mathscr{L}$ for some disjoint sets $\mathscr{K}, \mathscr{L}$.

The projection maps $\mathrm{pr}_{i}: V_{i+1} \rightarrow V_{i}$ induce maps $\mathrm{pr}_{i}^{*}: K\left[V_{i}\right] \rightarrow K\left[V_{i+1}\right]$ which are injective and degree-preserving. We will see that, for many of the maps $\mathrm{pr}_{i}$ we will encounter, the map $\operatorname{pr}_{i}^{*}$ is also grad-preserving. Since $X_{i+1}$ projects into $X_{i}$, we have $\operatorname{pr}_{i}^{*}\left(I\left(X_{i}\right)\right) \subseteq I\left(X_{i+1}\right)$. So $f$ induces non-zero elements $g_{j} \in I\left(X_{j}\right)$ of degree $d$ for all $j>i$. 
Let $A: K^{k} \rightarrow G_{j}$ be a polynomial map such that the map

$$
\begin{aligned}
K^{k} & \rightarrow G_{j} \\
\Lambda & \mapsto A(\Lambda)^{-1}
\end{aligned}
$$

is polynomial as well. Then $A(\Lambda) \cdot g_{j} \in I\left(X_{j}\right)$ for all $\Lambda \in K^{k}$ and therefore linear combinations of such elements also lie in $I\left(X_{j}\right)$. Note that we can view $A(\Lambda) \cdot g_{j}$ as a polynomial in the entries of $\Lambda$ whose coefficients are elements of $K\left[V_{j}\right]$. Let $R$ be a $K$-algebra and $h \in R[x]$ a polynomial. Then, since the field $K$ is infinite, one sees using a Vandermonde matrix that the coefficients of $h$ are contained in the $K$-span of $\{h(\lambda) \mid \lambda \in K\}$. Applying this fact $k$ times, we see that all the coeffiecients of $A(\Lambda) \cdot g_{j}$ lie in $\operatorname{span}\left(A(\Lambda) \cdot g_{j} \mid \Lambda \in K^{k}\right) \subseteq I\left(X_{j}\right)$.

We will let $f_{j}$ be a certain one of these coefficients. We have $\operatorname{deg}\left(f_{j}\right) \leq d$ by construction and we will choose $A$ in such a way that $f_{j}$ is "off-diagonal". We will see that $f_{j}$ is obtained from $g_{j}$ by substituting variables into the top-graded part of $g_{j}$ with respect to the right grading (in most cases deg or grad). Since the polynomial $g_{j}$ is non-zero, so is its top-graded part with respect to any grading. So it then suffices to check that this top-graded part does not become zero after the substitution. In the cases where is this not obvious, it will follow from a lemma stating that a certain morphism is dominant.

2.4. Using knowledge about stable closed subsets of the "off-diagonal" part. The space $V_{j}$ consists of matrices. When we have an "off-diagonal" polynomial which is contained in $I\left(X_{j}\right)$, we know that the projection $Y$ of $X_{j}$ onto some offdiagonal submatrix cannot form a dense subset of the projection $W$ of the whole of $V_{j}$. We then give $W$ the structure of a representation such that $Y$ is stable and use the fact the we know that the ideal of $Y$ contains a non-zero polynomial of degree at most $d$ to find conditions that hold for all elements of $Y$. These in turn give conditions that must hold for all elements of $X_{j}$, which will be enough to prove that $X$ is $G$-Noetherian.

\section{Limits OF CLASSicAl groups OF TYPE A}

In this section, we let $G$ be the direct limit of a sequence

$$
\mathrm{SL}_{n_{1}} \stackrel{l_{1}}{\longleftrightarrow} \mathrm{SL}_{n_{2}} \stackrel{\iota_{2}}{\longleftrightarrow} \mathrm{SL}_{n_{3}} \stackrel{l_{3}}{\longleftrightarrow \ldots}
$$

of diagonal embeddings given by

$$
\begin{aligned}
\iota_{i}: \mathrm{SL}_{n_{i}} & \hookrightarrow \mathrm{SL}_{n_{i+1}} \\
A & \mapsto \operatorname{Diag}\left(A, \ldots, A, A^{-T}, \ldots, A^{-T}, I_{z_{i}}\right)
\end{aligned}
$$

with $l_{i}$ blocks $A$ and $r_{i}$ blocks $A^{-T}$ for some $l_{i} \in \mathbb{N}$ and $r_{i}, z_{i} \in \mathbb{Z}_{\geq 0}$ with $l_{i} \geq r_{i}$. We let $V$ be the inverse limit of the sequence

$$
\mathfrak{g l}_{n_{1}} / \operatorname{span}\left(I_{n_{1}}\right) \longleftarrow \mathfrak{g l}_{n_{2}} / \operatorname{span}\left(I_{n_{2}}\right) \longleftarrow-\mathfrak{g l}_{n_{3}} / \operatorname{span}\left(I_{n_{3}}\right) \longleftarrow \leftarrow \ldots
$$


where the maps are given by

$$
\left(\begin{array}{ccccccc}
P_{11} & \ldots & P_{1 l_{i}} & \bullet & \ldots & \bullet & \bullet \\
\vdots & & \vdots & \vdots & & \vdots & \vdots \\
P_{l_{1} 1} & \ldots & P_{l_{l} l_{i}} & \bullet & \ldots & \bullet & \bullet \\
\bullet & \ldots & \bullet & Q_{11} & \ldots & Q_{1 r_{i}} & \bullet \\
\vdots & & \vdots & \vdots & & \vdots & \vdots \\
\bullet & \ldots & \bullet & Q_{r_{i} 1} & \ldots & Q_{r_{i} r_{i}} & \bullet \\
\bullet & \ldots & \bullet & \bullet & \ldots & \bullet & \bullet
\end{array}\right) \quad \bmod I_{n_{i+1}} \mapsto \sum_{k=1}^{l_{i}} P_{k k}-\sum_{\ell=1}^{r_{i}} Q_{\ell \ell}^{T} \bmod I_{n_{i}} .
$$

Here each $\bullet$ represents some matrix of the appropriate size. Our goal is to prove that the inverse limit $V$ of this sequence is G-Noetherian.

Take $\alpha=\#\left\{i \mid l_{i}>1\right\}, \beta=\#\left\{i \mid r_{i}>0\right\}, \gamma=\#\left\{i \mid z_{i}>0\right\} \in \mathbb{Z}_{\geq 0} \cup\{\infty\}$. Then we have $\alpha+\beta+\gamma=\infty$, since $G$ is assumed to be infinite-dimensional. Based on $\alpha, \beta, \gamma$ we distinguish the following cases:

(1) $\alpha+\beta<\infty$;

(2) $\alpha+\beta=\gamma=\infty$;

(3a) $\beta=\infty, \gamma<\infty$ and $\operatorname{char}(K) \neq 2$ or $2 \nmid n_{i}$ for all $i \gg 0$;

(3b) $\beta=\infty, \gamma<\infty$, $\operatorname{char}(K)=2$ and $2 \mid n_{i}$ for all $i \gg 0$;

(4a) $\beta+\gamma<\infty$ and $\operatorname{char}(K) \nmid n_{i}$ for all $i \gg 0$; and

(4b) $\beta+\gamma<\infty$ and $\operatorname{char}(K) \mid n_{i}$ for all $i \gg 0$.

Note here that if $\gamma<\infty$, then $n_{i} \mid n_{i+1}$ for all $i \gg 0$. Denote the element of $V$ representated by the sequence of zero matrices by 0 .

Theorem 13. The space $V$ is G-Noetherian. Any G-stable closed subset of $V$ is a finite union of irreducible G-stable closed subsets. The irreducible G-stable closed subsets of $V$ are $\{0\}$ and $V$ together with

$$
\left\{\left(P_{i} \bmod I_{n_{i}}\right)_{i} \in V \mid \forall i \gg 0: \operatorname{rk}\left(P_{i}, I_{n_{i}}\right) \leq k\right\}
$$

for $k \in \mathbb{N}$ in case (1) and together with

$$
\left\{\left(P_{i} \bmod I_{n_{i}}\right)_{i} \in V \mid \forall i \gg 0: \operatorname{tr}\left(P_{i}\right)=\mu\right\}
$$

for $\mu \in K$ in cases ( $3 b)$ and ( $4 b)$.

Here we call a closed subset $X \subseteq V$ irreducible when the following condition holds: if $X=Y \cup Z$ for (not necessarily $G$-stable) closed subsets $Y, Z \subseteq X$, then $X=Y$ or $X=Z$. The following proposition expresses the irreduciblility of a closed subset of $V$ in terms of the closures of its projections.

Proposition 14. Let

$$
W_{1} \longleftarrow W_{2} \longleftarrow W_{3} \longleftarrow \ldots
$$

be a sequence of finite-dimensional vector spaces with inverse limit $W$. Let $X \subseteq W$ be a closed subset and let $X_{i}$ be the closure of the projection of $X$ to $W_{i}$. Then the following are equivalent:

(1) $X$ is irreducible.

(2) $X_{i}$ is irreducible for all $i \geq 1$.

(3) $X_{i}$ is irreducible for all $i \gg 0$. 
Proof. Suppose that $X_{i}$ is reducible for some $i \in \mathbb{N}$. Then $X_{i}=Y \cup Z$ for some closed subsets $Y, Z \subsetneq X_{i}$. In this case, we see that

$$
X=\left(\operatorname{pr}_{i}^{-1}(Y) \cap X\right) \cup\left(\operatorname{pr}_{i}^{-1}(Z) \cap X\right), \quad \operatorname{pr}_{i}^{-1}(Y) \cap X, \operatorname{pr}_{i}^{-1}(Z) \cap X \subsetneq X
$$

and so $X$ is reducible. This establishes (1) $\Rightarrow(2)$. The implication (2) $\Rightarrow(3)$ is trivial. So next, if $X=Y \cup Z$ for some closed subsets $Y, Z \subsetneq X$ with closures $Y_{i}, Z_{i}$ in $W_{i}$, then $X_{i}=Y_{i} \cup Z_{i}$ for all $i \in \mathbb{N}$ and $Y_{i}, Z_{i} \subsetneq X_{i}$ for all $i \gg 0$. So in this case, we see that $X_{i}$ is reducible for $i \gg 0$.

3.1. The case $\alpha+\beta<\infty$. By replacing

$$
\mathrm{SL}_{n_{1}} \stackrel{\iota_{1}}{\longleftrightarrow} \mathrm{SL}_{n_{2}} \stackrel{\iota_{2}}{\longleftrightarrow} \mathrm{SL}_{n_{3}} \stackrel{\iota_{3}}{\longleftrightarrow \ldots}
$$

with some infinite subsequence, we may assume that $\left(l_{i}, r_{i}\right)=(1,0)$ and $z_{i}>0$ for all $i \in \mathbb{N}$. Then, by replacing the sequence by a supersequence, we may assume that $n_{i}=i$ and $z_{i}=1$ for all $i \in \mathbb{N}$. So we consider the inverse limit $V=\mathfrak{g l}_{\infty} / \operatorname{span}\left(I_{\infty}\right)$ of the sequence

$$
\mathrm{gl}_{1} / \operatorname{span}\left(I_{1}\right) \nVdash \quad \mathrm{gl}_{2} / \operatorname{span}\left(I_{2}\right) \longleftarrow-\mathrm{gl}_{3} / \operatorname{span}\left(I_{3}\right) \nVdash \ldots
$$

acted on by the group $G=\mathrm{SL}_{\infty}$. The $\mathrm{SL}_{\infty}$-stable closed subsets of $\mathfrak{g l}_{\infty} / \operatorname{span}\left(I_{\infty}\right)$ correspond one-to-one to the $\mathrm{SL}_{\infty}$-stable closed subsets $X$ of $\mathfrak{g l}_{\infty}$ such that

$$
X+\operatorname{span}\left(I_{\infty}\right)=X .
$$

Theorem 5 therefore tells us exactly what the $G$-stable closed subsets of $V$ are. The next proposition shows that Theorem 5 implies case (1) of Theorem 13 .

Proposition 15. Let $P_{1}, \ldots, P_{k}$ be elements of $\mathrm{gl}_{\infty}$. Then we have

$$
\operatorname{rk}\left(P_{1}, \ldots, P_{k}\right)=\sup \left\{\operatorname{rk}\left(\operatorname{pr}_{n}\left(P_{1}\right), \ldots, \operatorname{pr}_{n}\left(P_{k}\right)\right) \mid n \in \mathbb{N}\right\} .
$$

Proof. We have $\operatorname{rk}\left(\operatorname{pr}_{n}\left(P_{1}\right), \ldots, \operatorname{pr}_{n}\left(P_{k}\right)\right) \leq \operatorname{rk}\left(\mu_{1} P_{1}+\cdots+\mu_{k} P_{k}\right)$ for all $n \in \mathbb{N}$ and $\left(\mu_{1}: \cdots: \mu_{k}\right) \in \mathbb{P}^{k-1}$. So

$$
r:=\sup \left\{\operatorname{rk}\left(\operatorname{pr}_{n}\left(P_{1}\right), \ldots, \operatorname{pr}_{n}\left(P_{k}\right)\right) \mid n \in \mathbb{N}\right\} \leq \operatorname{rk}\left(P_{1}, \ldots, P_{k}\right)
$$

with equality when $r=\infty$. Suppose that $r<\infty$ and consider the descending chain

$$
Y_{1} \supseteq Y_{2} \supseteq Y_{3} \supseteq Y_{4} \supseteq \ldots
$$

of closed subsets of $\mathbb{P}^{k-1}$ defined by

$$
Y_{n}=\left\{\left(\mu_{1}: \cdots: \mu_{k}\right) \in \mathbb{P}^{k-1} \mid \operatorname{rk}\left(\mu_{1} \operatorname{pr}_{n}\left(P_{1}\right)+\cdots+\mu_{k} \operatorname{pr}_{n}\left(P_{k}\right)\right) \leq r\right\} .
$$

By construction, each $Y_{n}$ is non-empty. And by the Noetherianity of $\mathbb{P}^{k-1}$, the chain stabilizes. Let $\left(\mu_{1}: \cdots: \mu_{k}\right) \in \mathbb{P}^{k-1}$ be an element contained in $Y_{n}$ for all $n \in \mathbb{N}$. Then we see that $\operatorname{rk}\left(P_{1}, \ldots, P_{k}\right) \leq \operatorname{rk}\left(\mu_{1} P_{1}+\cdots+\mu_{k} P_{k}\right) \leq r$.

So we proceed to prove Theorem 5 . The following proposition, which is due to Jan Draisma, connects the tuple rank of a matrix $P$ with the identity matrix to the rank of off-diagonal submatrices of matrices similar to $P$.

Proposition 16. Let $k, m, n \in \mathbb{Z}_{\geq 0}$ be such that $n \geq 2 m \geq 2(k+1)$, let $\mathscr{K}, \mathscr{L}$ be disjoint subsets of $[n]$ of size $m$ and let $P$ be an $n \times n$ matrix. Then $\operatorname{rk}\left(P, I_{n}\right) \leq k$ if and only if the submatrix $Q \mathscr{K}, \mathscr{L}$ of $Q$ has rank at most $k$ for every $Q \sim P$. 
Proof. Suppose that $\operatorname{rk}\left(P, I_{n}\right) \leq k$. Let $Q \sim P$ be a similar matrix. Then $\operatorname{rk}\left(Q, I_{n}\right) \leq k$. So since $\mathscr{K} \cap \mathscr{L}=\emptyset$ and the off-diagonal entries of $Q$ and $Q-\lambda I_{n}$ are equal for all $\lambda \in K$, we see that $\operatorname{rk}\left(Q_{\mathscr{K}, \mathscr{L}}\right) \leq k$.

Suppose that the submatrix $Q_{\mathscr{K}, \mathscr{L}}$ has rank at most $k$ for every $Q \sim P$. Then this statement still holds when we replace $\mathscr{K}$ and $\mathscr{L}$ by subsets of themselves of size $k+1$. This reduces the proposition to the case $m=k+1$. Now the statement we want to prove is implied by the following coordinate-free version:

$\left({ }^{*}\right)$ Let $V$ be a vector space of dimension $n$ and let $\varphi: V \rightarrow V$ be an endomorphism. If the induced map $\varphi: W \rightarrow V / W$ has a non-trivial kernel for all $(k+1)$-dimensional subspaces $W$ of $V$, then $\varphi$ has an eigenvalue of geometric multiplicity at least $n-k$.

Indeed, taking $\varphi: K^{n} \rightarrow K^{n}$ the endomorphism corresponding to $P$ and $W \subseteq K^{n}$ a $(k+1)$-dimensional subspace, we can first replace $P$ be a matrix $Q \sim P$ to get $W=K^{k+1} \times\{0\}$. Since $Q$ is similar to all its conjugates by permutation matrices, we know that $\operatorname{det}\left(Q_{\mathcal{K}, \mathscr{L}}\right)=0$ for all disjoint subsets of $\mathscr{K}, \mathscr{L} \subseteq[n]$ of size $m$. Hence $Q_{[n] \backslash k+1],[k+1]}$ has rank at most $k$. So the induced map $W \rightarrow V / W$ has a non-trivial kernel. We conclude from $\left({ }^{*}\right)$ that

$$
\operatorname{rk}\left(P-\lambda I_{n}\right)=\operatorname{rk}\left(Q-\lambda I_{n}\right) \leq n-(n-k)=k
$$

for some $\lambda \in K$. So $\operatorname{rk}\left(P, I_{n}\right) \leq k$.

To prove $\left({ }^{*}\right)$, consider the incidence variety

$$
Z=\left\{(W,[v]) \in \mathrm{Gr}_{k+1}(V) \times \mathbb{P}(V) \mid v, \varphi(v) \in W\right\}
$$

and let $\pi_{1}, \pi_{2}$ be the projections from $Z$ to the Grassmannian $\mathrm{Gr}_{k}(V)$ and to $\mathbb{P}(V)$. By assumption $\pi_{1}$ is surjective. So we have

$$
\operatorname{dim} Z \geq \operatorname{dim}\left(\mathrm{Gr}_{k+1}(V)\right)=(k+1)(n-k-1) .
$$

On the other hand, let $v \in V \backslash\{0\}$ be a non-eigenvector of $\varphi$. Then $\pi_{1}\left(\pi_{2}^{-1}([v])\right)$ consists of all $W \in \mathrm{Gr}_{k+1}(V)$ containing $\operatorname{span}(v, \varphi(v))$ and these form the Grassmannian $\mathrm{Gr}_{k-1}(V / \operatorname{span}(v, \varphi(v)))$ of dimension $(k-1)(n-k-1)$. Thus the union of the fibres $\pi_{2}^{-1}([v])$ for $v$ not an eigenvector of $\varphi$ has dimension at most

$$
(k-1)(n-k-1)+\operatorname{dim}(\mathbb{P}(V))=(k+1)(n-k-1)+2 k+1-n .
$$

This dimension is strictly smaller than $\operatorname{dim}(Z)$. Let $v$ be an eigenvector of $\varphi$. Then $\pi_{1}\left(\pi_{2}^{-1}([v])\right)$ consists of all $W \in \mathrm{Gr}_{k+1}(V)$ with $v \in W$ and these form the Grassmannian $\mathrm{Gr}_{k}(V / \operatorname{span}(v))$ of dimension $k(n-k-1)$. So we see that the union of the eigenspaces of $\varphi$ must have dimension at least $\operatorname{dim}(Z)-k(n-k-1)+1 \geq n-k$. Hence some eigenspace of $\varphi$ must have dimension al least $n-k$.

Definition 17. For $n \in \mathbb{N}$, we call a polynomial $f \in K\left[\mathrm{gl}_{n}\right]$ off-diagonal if

$$
f \in K\left[p_{k \ell} \mid k \in \mathscr{K}, \ell \in \mathscr{L}\right]
$$

for some disjoint subsets $\mathscr{K}, \mathscr{L} \subset[n]$ of size $m \leq(n-1) / 2$.

Lemma 18. Let $n \in \mathbb{N}$ be an integer, let $Y$ be an $\mathrm{SL}_{n}$-stable closed subset of $\mathfrak{g l}_{n}$ and suppose that $I(Y)$ contains a non-zero off-diagonal polynomial $f$. Then $\operatorname{rk}\left(P, I_{n}\right)<\operatorname{deg}(f)$ for all $P \in Y$. 
Proof. Let $\mathscr{K}, \mathscr{L} \subset[n]$ be disjoint subsets of size $m \leq n / 2$ and let

$$
f \in K\left[p_{k \ell} \mid k \in \mathscr{K}, \ell \in \mathscr{L}\right] \cap I(Y)
$$

be a non-zero element. If $m=0$, then $f$ is constant and $Y=\emptyset$. So in particular, $\operatorname{rk}\left(P, I_{n}\right)<\operatorname{deg}(f)$ for all $P \in Y$. For $m>0$, let $Z$ be the closure of the set

$$
\left\{\left(y_{k \ell}\right)_{k \in \mathcal{K}, \ell \in \mathscr{L}} \mid\left(y_{k \ell}\right)_{k, \ell} \in Y\right\}
$$

in $\mathfrak{g l}_{m}$. Then $f \in I(Z)$. By conjugating with with \pm 1 times a permutation matrix, we may assume that $\mathscr{K}=[m]$ and $\mathscr{L}=[2 m] \backslash[m]$. Now consider the map

$$
\begin{aligned}
\mathrm{GL}_{m} \times \mathrm{GL}_{m} & \rightarrow \mathrm{SL}_{n} \\
(A, B) & \mapsto \operatorname{Diag}\left(A, B, I_{n-2 m-1}, \operatorname{det}(A B)^{-1}\right) .
\end{aligned}
$$

Since $Y$ is $\mathrm{GL}_{m} \times \mathrm{GL}_{m}$-stable, we see that $Z$ is closed under $\mathrm{GL}_{m} \times \mathrm{GL}_{m}$ acting by left and right multiplication. So $Z$ must consist of all matrices of rank at most $\ell$ for some $\ell \leq m$. Since $f \in I(Z)$, we see that $\ell<\min (m, \operatorname{deg}(f))$. So by Proposition 16, we see that $Y$ consists of matrices $P$ such that $\operatorname{rk}\left(P, I_{n}\right)<\min (m, \operatorname{deg}(f)) \leq \operatorname{deg}(f)$.

Remark 19. Let $Y$ be a SL ${ }_{n}$-stable closed subset of $\mathfrak{g l}_{n} / \operatorname{span}\left(I_{n}\right)$. Then we can apply Lemma 18 to $Y$ by considering its inverse image in $\mathrm{gl}_{n}$. So if $I(Y)$ contains a non-zero off-diagonal polynomial $f$, then $\operatorname{rk}\left(P, I_{n}\right)<\operatorname{deg}(f)$ for all $\left(P \bmod I_{n}\right) \in Y$.

Let $X$ be a proper $\mathrm{SL}_{\infty}$-stable closed subset of $\mathrm{gl}_{\infty}$. Denote the closure of the projection of $X$ to $\mathfrak{g l}_{n}$ by $X_{n}$ and let $I\left(X_{n}\right) \subseteq K\left[\mathfrak{g l}_{n}\right]$ be its corresponding ideal.

Lemma 20. Let $m$ be a positive integer and suppose that $I\left(X_{m}\right)$ contains a non-zero polynomial $f$. Then $r k\left(P, I_{\infty}\right)<\operatorname{deg}(f)$ for all $P \in X$.

Proof. Note that the morphism $X_{n} \rightarrow X_{m}$ is dominant for all positive integers $m \leq n$.

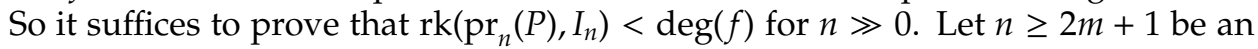
integer. Then $f$ induces the element

$$
g=\left(\left(\begin{array}{lll}
P & Q & \bullet \\
R & S & \bullet \\
\bullet & \bullet & \bullet
\end{array}\right) \mapsto f(P)\right)
$$

of $I\left(X_{n}\right)$ where $P, Q, R, S \in \mathfrak{g l}_{m}$. This allows us to assume that $\operatorname{deg}(f)<m$ without loss of generality. For $\lambda \in K$, consider the matrix

$$
A(\lambda)=\left(\begin{array}{ccc}
I_{m} & \lambda I_{m} & \\
& I_{m} & \\
& & I_{n-2 m}
\end{array}\right) \in \mathrm{SL}_{n} .
$$

We have

$$
A(\lambda)\left(\begin{array}{lll}
P & Q & \bullet \\
R & S & \bullet \\
\bullet & \bullet & \bullet
\end{array}\right) A(\lambda)^{-1}=\left(\begin{array}{ccc}
P+\lambda R & Q+\lambda(S-P)-\lambda^{2} R & \bullet \\
R & S-\lambda R & \bullet \\
\bullet & \bullet & \bullet
\end{array}\right)
$$

for all $\lambda \in K$. So we see that if we let $A(\lambda)$ act on $g$, we obtain the element

$$
h_{\lambda}=\left(\left(\begin{array}{ccc}
P & Q & \bullet \\
R & S & \bullet \\
\bullet & \bullet & \bullet
\end{array}\right) \mapsto f(P+\lambda R)\right)
$$

of $I\left(X_{n}\right)$. Let $k+1$ be the degree of $f$ and let $f_{k+1}$ be the homogeneous part of $f$ of degree $k+1$. Then the homogeneous part of $h_{\lambda}$ of degree $k+1$ in $\lambda$ equals the 
polynomial $\lambda^{k+1} f_{k+1}(R)$. Since the field $K$ is infinite, the polynomial $f_{k+1}(R)$ is a linear combination of the $h_{\lambda}$. Hence $f_{k+1}(R) \in I\left(X_{n}\right)$. So $\operatorname{rk}\left(P, I_{n}\right)<\operatorname{deg}(f)$ for all $P \in X_{n}$ by Lemma 18 and therefore $\operatorname{rk}\left(P, I_{\infty}\right)<\operatorname{deg}(f)$ for all $P \in X$.

Lemma 21. Let $k<n$ be non-negative integers and let $P \in \mathfrak{g I}_{2 n}$ and $Q \in \mathfrak{g I}_{n}$ be matrices with $\operatorname{rk}(P)=k$ and $\operatorname{rk}(Q) \leq k$. Then $P$ is similar to

$$
\left(\begin{array}{cc}
Q & Q_{12} \\
Q_{21} & Q_{22}
\end{array}\right)
$$

for some $Q_{12}, Q_{21}, Q_{22} \in \mathfrak{g I}_{n}$.

Proof. First note that $\operatorname{rk}\left(P, I_{2 n}\right)=2 n-\operatorname{dim} \operatorname{ker}(P)=k$, since 0 has the highest geometric multiplicity among all eigenvalues of $P$. Since $2(k+1) \leq 2 n$, it follows by Proposition 16 that

$$
P \sim\left(\begin{array}{ll}
\bullet & \bullet \\
R & \bullet
\end{array}\right)
$$

for some matrix $R \in \mathfrak{g I}_{n}$ with $\operatorname{rk}(R)=k$. By conjugating the latter matrix with $\operatorname{Diag}\left(g, I_{n}\right)$ for some $g \in \mathrm{GL}_{n}$ such that $g \operatorname{ker}(R) \subseteq \operatorname{ker}(Q)$, we see that

$$
\left(\begin{array}{ll}
\bullet & \bullet \\
R & \bullet
\end{array}\right) \sim\left(\begin{array}{cc}
\bullet & \bullet \\
R^{\prime} & \bullet
\end{array}\right)
$$

for some matrix $R^{\prime} \in \mathfrak{g l}_{n}$ with $\operatorname{rk}\left(R^{\prime}\right)=k$ and $\operatorname{ker}\left(R^{\prime}\right) \subseteq \operatorname{ker}(Q)$. This means that $Q=S R^{\prime}$ for some $S \in \mathfrak{g l}_{n}$. Since both $R^{\prime}$ and any matrix similar to $P$ have rank $k$, we see that the matrix on the right must be of the form

$$
\left(\begin{array}{cc}
T R^{\prime} & \bullet \\
R^{\prime} & \bullet
\end{array}\right)
$$

for some $T \in \mathfrak{g l}_{n}$. Now note that the matrix

$$
\left(\begin{array}{cc}
I_{n} & S-T \\
0 & I_{n}
\end{array}\right)\left(\begin{array}{cc}
T R^{\prime} & \bullet \\
R^{\prime} & \bullet
\end{array}\right)\left(\begin{array}{cc}
I_{n} & T-S \\
0 & I_{n}
\end{array}\right)=\left(\begin{array}{cc}
S R^{\prime} & \bullet \\
R^{\prime} & \bullet
\end{array}\right)=\left(\begin{array}{cc}
Q & \bullet \\
R^{\prime} & \bullet
\end{array}\right)
$$

is similar to $P$ and of the form we want.

Proposition 22. Let $P \in \mathfrak{g l}_{\infty}$ be an element. Then either the orbit of $P$ is dense in $\mathfrak{g l}_{\infty}$ or $k=\operatorname{rk}\left(P-\lambda I_{\infty}\right)<\infty$ for some unique $\lambda \in K$. In the second case, the closure of the orbit of $P$ equals the irreducible closed subset $\left\{Q \in \mathfrak{g l}_{\infty} \mid \operatorname{rk}\left(Q-\lambda I_{\infty}\right) \leq k\right\}$ of $\mathfrak{g l}_{\infty}$.

Proof. Let $X$ be the closure of the orbit of $P$. Then either $X=\mathfrak{g l}_{\infty}$ or $\operatorname{rk}\left(P, I_{\infty}\right)=k$ for some $k \in \mathbb{Z}_{\geq 0}$ by Lemma20 In the second case, we see that $\operatorname{rk}\left(P-\lambda I_{\infty}\right)=k$ for some unique $\lambda \in K$ by Remark 7 . Our goal is to prove that $X=\left\{Q \in \mathrm{gl}_{\infty} \mid \operatorname{rk}\left(Q-\lambda I_{\infty}\right) \leq k\right\}$. Using the $\mathrm{SL}_{\infty}$-equivariant affine isomorphism

$$
\begin{aligned}
\mathfrak{g l}_{\infty} & \rightarrow \mathfrak{g l}_{\infty} \\
Q & \mapsto Q-\lambda I_{\infty}
\end{aligned}
$$

we may assume that $\lambda=0$ and hence that $k=\operatorname{rk}(P)$ is finite. It suffices to prove that

$$
\operatorname{pr}_{n}\left(\left\{Q \in \mathrm{gl}_{\infty} \mid \operatorname{rk}(Q) \leq k\right\}\right)=\left\{Q \in \mathfrak{g l}_{n} \mid \operatorname{rk}(Q) \leq k\right\}=\operatorname{pr}_{n}\left(\mathrm{SL}_{\infty} \cdot P\right)
$$

for all $n \gg 0$ since the middle set is irreducible. See Proposition 14 The inclusions

$$
\operatorname{pr}_{n}\left(\mathrm{SL}_{\infty} \cdot P\right) \subseteq \operatorname{pr}_{n}\left(\left\{Q \in \mathrm{gl}_{\infty} \mid \operatorname{rk}(Q) \leq k\right\}\right) \subseteq\left\{Q \in \mathrm{gl}_{n} \mid \operatorname{rk}(Q) \leq k\right\}
$$


are clear for all $n \in \mathbb{N}$. Let $n>k$ be an integer such that the rank of $\operatorname{pr}_{2 n}(P)$ equals $k$. Then

$$
\left\{Q \in \mathrm{gl}_{n} \mid \operatorname{rk}(Q) \leq k\right\} \subseteq \operatorname{pr}_{n}\left(\mathrm{SL}_{2 n} \cdot \mathrm{pr}_{2 n}(P)\right) \subseteq \operatorname{pr}_{n}\left(\mathrm{SL}_{\infty} \cdot P\right)
$$

by Lemma 21, So indeed $\operatorname{pr}_{n}\left(\left\{Q \in \mathfrak{g l}_{\infty} \mid \operatorname{rk}(Q) \leq k\right\}\right)=\operatorname{pr}_{n}\left(\mathrm{SL}_{\infty} \cdot P\right)$ for all $n \gg 0$.

Lemma 23. Let $m$ be a positive integer and suppose that $I\left(X_{m}\right)$ contains a non-zero polynomial $f$ with $\operatorname{deg}(f)<m$. Let $g(t)=f\left(t I_{m}\right) \in K[t]$ be the restriction of $f$ to $\operatorname{span}\left(I_{m}\right)$. Then $X$ is contained in

$$
\bigcup_{\lambda}\left\{Q \in \mathrm{gl}_{\infty} \mid \operatorname{rk}\left(Q-\lambda I_{\infty}\right)<\operatorname{deg}(f)\right\}
$$

where $\lambda \in K$ ranges over the zeros of $g$.

Proof. Let $P$ be an element of $X$. Since $f$ is non-zero, we know that $X$ is a proper $\mathrm{SL}_{\infty}$-stable closed subset of $\mathfrak{g l}_{\infty}$. Hence the orbit of $P$ cannot be dense in $\mathfrak{g l}_{\infty}$. So $k=\operatorname{rk}\left(P-\lambda I_{\infty}\right)<\operatorname{deg}(f)$ for some $\lambda \in K$ by Lemma 20. This $\lambda$ is unique and the closure of the orbit of $P$ equals $\left\{Q \in \mathfrak{g l}_{\infty} \mid \operatorname{rk}\left(Q-\lambda I_{\infty}\right) \leq k\right\}$ by Proposition 22 So we see that $\lambda I_{\infty}$ is an element of $X$. So $\lambda I_{m}$ is an element of $X_{m}$ and hence $g(\lambda)=f\left(\lambda I_{m}\right)=0$. We see that for all $P \in X$ there is a $\lambda \in K$ with $g(y)=0$ such that

$$
P \in\left\{Q \in \mathfrak{g l}_{\infty} \mid \operatorname{rk}\left(Q-\lambda I_{\infty}\right)<\operatorname{deg}(f)\right\} .
$$

Proposition 24. Either the $\mathrm{SL}_{\infty}$-stable closed subset $\operatorname{span}\left(I_{\infty}\right)$ of $\mathrm{gl}_{\infty}$ is contained in $\mathrm{X}$ or there exist $\lambda_{1}, \ldots, \lambda_{\ell} \in K$ and $k_{1}, \ldots, k_{\ell} \in \mathbb{Z}_{\geq 0}$ such that

$$
X=\bigcup_{i=1}^{\ell}\left\{Q \in \mathfrak{g l}_{\infty} \mid \operatorname{rk}\left(Q-\lambda_{i} I_{\infty}\right) \leq k_{i}\right\} .
$$

Proof. Assume that $\operatorname{span}\left(I_{\infty}\right)$ is not contained in $X$. Then, for some $m \in \mathbb{N}, X_{m}$ is a proper subset of $\mathfrak{g l}_{m}$ that does not contain span $\left(I_{m}\right)$. The ideal $I\left(X_{m}\right)$ must contain a non-zero polynomial $f$ such that the polynomial $g(t)=f\left(t I_{m}\right) \in K[t]$ is non-zero. By Lemma 23, we see that $X$ is contained in

$$
\bigcup_{\lambda}\left\{Q \in \mathfrak{g l}_{\infty} \mid \operatorname{rk}\left(Q-\lambda I_{\infty}\right)<\operatorname{deg}(f)\right\}
$$

where $\lambda \in K$ ranges over the finitely many zeros of $g$. Take

$$
\Lambda=\left\{\lambda \in K \mid g(\lambda)=0, \exists P \in X: \operatorname{rk}\left(P-\lambda I_{\infty}\right)<\operatorname{deg}(f)\right\}
$$

and take

$$
k_{\lambda}=\max \left\{\operatorname{rk}\left(P-\lambda I_{\infty}\right) \mid P \in X, \operatorname{rk}\left(P-\lambda I_{\infty}\right)<\infty\right\}
$$

for all $\lambda \in \Lambda$. Then we see that

$$
X=\bigcup_{\lambda \in \Lambda}\left\{Q \in \mathfrak{g l}_{\infty} \mid \operatorname{rk}\left(Q-\lambda I_{\infty}\right) \leq k_{\lambda}\right\}
$$

using Proposition 22

The proposition implies in particular that any descending chain of $\mathrm{SL}_{\infty}$-stable closed subsets of $\mathfrak{g l}_{\infty}$ stablizes as long as one of these subsets does not contain $\operatorname{span}\left(I_{\infty}\right)$. Next we will classify the subsets that do contain $\operatorname{span}\left(I_{\infty}\right)$. 
Proposition 25. Let $k$ be a non-negative integer. Then the $\mathrm{SL}_{\infty}$-stable subset

$$
\left\{P \in \mathrm{gl}_{\infty} \mid \operatorname{rk}\left(P, I_{\infty}\right) \leq k\right\}
$$

of $\mathrm{gl}_{\infty}$ is closed and irreducible.

Proof. Using Proposition 15, we see that

$$
\left\{P \in \mathfrak{g l}_{\infty} \mid \operatorname{rk}\left(P, I_{\infty}\right) \leq k\right\}
$$

its the inverse limit of its projections $\left\{P \in \mathfrak{g l}_{n} \mid \operatorname{rk}\left(P, I_{n}\right) \leq k\right\}$ onto $\mathfrak{g l}_{n}$. So it suffices to show that this is a closed irreducible subset of $\mathfrak{g l}_{n}$ for all $n \in \mathbb{N}$. See Proposition 14 The subset $\left\{P \in \mathfrak{g l}_{n} \mid \operatorname{rk}\left(P, I_{n}\right) \leq k\right\}$ is the inverse image of the subset

$$
Y=\left\{(P, Q) \in \mathrm{gl}_{n}^{2} \mid \operatorname{rk}(P, Q) \leq k\right\}
$$

under the map $\mathfrak{g l}_{n} \rightarrow \mathfrak{g l}_{n}^{2}, P \mapsto\left(P, I_{n}\right)$. The subset $Y$ is closed in $\mathfrak{g l}_{n}^{2}$ since it is the image of the closed subset

$$
\left\{\left(\left(\mu_{1}: \mu_{2}\right), P, Q\right) \in \mathbb{P}^{1} \times \mathfrak{g l}_{n}^{2} \mid \operatorname{rk}\left(\mu_{1} P+\mu_{2} Q\right) \leq k\right\}
$$

under the projection map along the complete variety $\mathbb{P}^{1}$. So $\left\{P \in \operatorname{gl}_{n} \mid \operatorname{rk}\left(P, I_{n}\right) \leq k\right\}$ is a closed subset of $\mathrm{gl}_{n}$. This subset is also the image of the map

$$
\begin{aligned}
\left\{Q \in \mathrm{gl}_{n} \mid \operatorname{rk}(Q) \leq k\right\} \times K & \rightarrow \mathrm{gl}_{n} \\
(Q, \lambda) & \mapsto Q+\lambda I_{n}
\end{aligned}
$$

and hence irreducible.

Proposition 26. Suppose that $X$ contains $\operatorname{span}\left(I_{\infty}\right)$. Then

$$
X=\left\{P \in \mathfrak{g l}_{\infty} \mid \operatorname{rk}\left(P, I_{\infty}\right) \leq k\right\} \cup Y
$$

for some non-negative integer $k$ and some $\mathrm{SL}_{\infty}$-stable closed subset $Y$ of $\mathrm{gl}_{\infty}$ that does not contain $\operatorname{span}\left(I_{\infty}\right)$.

Proof. Since $X$ is a proper subset of $\mathfrak{g l}_{\infty}$, we know that

$$
X \subseteq\left\{P \in \mathrm{gl}_{\infty} \mid \operatorname{rk}\left(P, I_{\infty}\right) \leq \ell\right\}
$$

for some $\ell \in \mathbb{Z}_{\geq 0}$ by Lemma 20. Let $k$ be the maximal non-negative integer such that

$$
\left\{P \in \mathrm{gl}_{\infty} \mid \operatorname{rk}\left(P, I_{\infty}\right) \leq k\right\} \subseteq X
$$

We will prove the statement by induction on the difference between $\ell$ and $k$.

Suppose that $\ell=k$. Then $X=\left\{P \in \mathfrak{g l}_{\infty} \mid \operatorname{rk}\left(P, I_{\infty}\right) \leq k\right\}$ and the statement holds. Now suppose that $\ell>k$ and let $Y^{\prime}$ be an $\mathrm{SL}_{\infty}$-stable closed subset of $\mathrm{gl}_{\infty}$ that does not contain $\operatorname{span}\left(I_{\infty}\right)$ such that

$$
X \cap\left\{P \in \mathrm{gl}_{\infty} \mid \operatorname{rk}\left(P, I_{\infty}\right) \leq \ell-1\right\}=\left\{P \in \mathfrak{g l}_{\infty} \mid \operatorname{rk}\left(P, I_{\infty}\right) \leq k\right\} \cup Y^{\prime} .
$$

Consider the set $Z=\left\{\lambda \in K \mid \exists P \in X: \operatorname{rk}\left(P-\lambda I_{\infty}\right)=\ell\right\}$ and fix an element $Q \in \mathfrak{g l}_{\infty}$ with $\operatorname{rk}(Q)=\ell$. By Proposition 22, we know for $\lambda \in K$ that $Q+\lambda I_{\infty} \in X$ if and only if $\lambda \in Z$. This shows that $Z$ is a closed subset of $K$. So either $Z=K$ or $Z$ is finite. If $Z=K$, then we see that $X$ contains all $P \in \mathfrak{g l}_{\infty}$ with $\operatorname{rk}\left(P, I_{\infty}\right) \leq \ell$ by Proposition 22. Since $\ell>k$, this is not true and hence $Z$ is finite. Take

$$
Y=Y^{\prime} \cup \bigcup_{\lambda \in Z}\left\{P \in \mathrm{gl}_{\infty} \mid \operatorname{rk}\left(P-\lambda I_{\infty}\right) \leq \ell\right\}
$$

Then we see that $X=\left\{P \in \mathfrak{g l}_{\infty} \mid \operatorname{rk}\left(P, I_{\infty}\right) \leq k\right\} \cup Y$. 
Proof of Theorem 5 Let $S$ be the set pairs $(k, f)$ where $k \in \mathbb{Z}_{\geq-1}$ and where $f: K \rightarrow$ $\mathbb{Z}_{\geq k}$ is a function such that $f^{-1}\left(\mathbb{Z}_{>k}\right)$ is finite. Define a partial ordering on $S$ by $(k, f) \leq(\ell, g)$ when $k \leq \ell$ and $f(\lambda) \leq g(\lambda)$ for all $\lambda \in K$. Then for all $(k, f) \in S$, the set $\{(k, g) \in S \mid(k, g) \leq(k, f)\}$ is finite. So any descending chain in $S$ stabilizes. For a proper $\mathrm{SL}_{\infty}$-stable closed subset $X$ of $\mathfrak{g l}_{\infty}$, let $k_{X}$ be the maximal integer such that $\left\{P \in \mathfrak{g l}_{\infty} \mid \operatorname{rk}\left(P, I_{\infty}\right) \leq k_{X}\right\} \subseteq X$ and let $f_{X}: K \rightarrow \mathbb{Z}_{\geq k}$ be the function sending $\lambda \in K$ to the maximal $k$ such that $\left\{P \in \mathrm{gl}_{\infty} \mid \operatorname{rk}\left(P-\lambda I_{\infty}\right) \leq k\right\} \subseteq X$. Then, by Propositions 24 and 26, we see that

$$
X=\left\{P \in \mathfrak{g l}_{\infty} \mid \operatorname{rk}\left(P, I_{\infty}\right) \leq k_{X}\right\} \cup \bigcup_{\lambda \in f_{X}^{-1}\left(\mathbb{Z}_{>k_{X}}\right)}\left\{P \in \mathfrak{g l}_{\infty} \mid \operatorname{rk}\left(P-\lambda I_{\infty}\right) \leq f_{X}(\lambda)\right\}
$$

and that the map $X \mapsto\left(k_{X}, f_{X}\right)$ is an order preserving bijection between the set of proper $\mathrm{SL}_{\infty}$-stable closed subsets of $\mathrm{gl}_{\infty}$ and $S$. Now consider a descending chain

$$
X_{1} \supseteq X_{2} \supseteq X_{3} \supseteq X_{4} \supseteq \ldots
$$

of $\mathrm{SL}_{\infty}$-stable closed subsets of $\mathfrak{g l}_{\infty}$. We get a descending chain

$$
\left(k_{X_{1}}, f_{X_{1}}\right) \geq\left(k_{X_{2}}, f_{X_{2}}\right) \geq\left(k_{X_{3}}, f_{X_{3}}\right) \geq\left(k_{X_{4}}, f_{X_{4}}\right) \geq \ldots
$$

in $S$ which must stabilize. Therefore the original chain also stabilizes. Hence $\mathrm{gl}_{\infty}$ is $\mathrm{SL}_{\infty}$-Noetherian. The irreducible $\mathrm{SL}_{\infty}$-stable closed subsets of $\mathfrak{g l}_{\infty}$ are as described in the theorem by Propositions 22, 24, 25, and 26.

Remark 27. The techniques used in the section can also be used to generalize Theorem 1.5 from $[\overline{D E}]$ to $G$-Noetherianity where $G=\left\{(g, g) \mid g \in \mathrm{GL}_{\infty}\right\}$. This generalization also follows from Theorem 1.2 of [ES].

3.2. The proof of the other cases. Now, we turn our attention to cases (2)-(4b) of Theorem 13 We start by proving some statements that are useful in multiple cases.

Lemma 28. Let $k, n$ be positive integers with $k \leq n$ and let $P \in \mathfrak{g l}_{n}$ be a matrix. Then $\operatorname{rk}(P)<k$ if and only if $\operatorname{det}\left(Q_{[k],[k]}\right)=0$ for all $Q \sim P$.

Proof. If $\operatorname{rk}(P)<k$, then $\operatorname{det}\left(Q_{[k],[k]}\right)=0$ for all $Q \sim P$. Suppose that $\operatorname{det}\left(Q_{[k],[k]}\right)=0$ for all $Q \sim P$. Note that $\operatorname{rk}(P)<k$ if and only if $\operatorname{det}\left(P_{\mathscr{K}, \mathscr{L}}\right)=0$ for all subsets $\mathscr{K}, \mathscr{L} \subset[n]$ of size $k$. One can prove this using reverse induction of the size of $\mathscr{K} \cap \mathscr{L}$. If $\mathscr{K}=\mathscr{L}$, then $P_{\mathscr{K}, \mathscr{L}}=Q_{[k],[k]}$ for some matrix $Q \sim P$ obtained from $P$ by conjugating with a permutation matrix. So $\operatorname{det}\left(P_{\mathscr{K}, \mathscr{L}}\right)=0$. For $|\mathscr{K} \cap \mathscr{L}|<k$, we take $i \in \mathscr{K} \backslash \mathscr{L}, j \in \mathscr{L} \backslash \mathscr{K}$ and $\mathscr{K}^{\prime}=\{j\} \cup \mathscr{K} \backslash\{i\}$ and note that, since $\left|\mathscr{K}^{\prime} \cap \mathscr{L}\right|>|\mathscr{K} \cap \mathscr{L}|$,

$$
\operatorname{det}\left(P_{\mathscr{K}, \mathscr{L}}\right)= \pm \operatorname{det}\left(P_{\mathscr{K}^{\prime}, \mathscr{L}}\right) \pm \operatorname{det}\left(Q_{\mathscr{K}^{\prime}, \mathscr{L}}\right)=0
$$

where $Q \sim P$ is the matrix obtained from $P$ by adding row $i$ to row $j$ and substracting column $j$ from column $i$.

Lemma 29. Let $k, \ell, n \in \mathbb{N}$ be integers with $n \geq 6 k$ and $\ell \geq 2$ and let $P_{1}, \ldots, P_{\ell} \in \mathfrak{g l}_{n}$ be matrices of rank $k$. Then there exist $Q_{1} \sim P_{1}, \ldots, Q_{\ell} \sim P_{\ell}$ such that

$$
k<\operatorname{rk}\left(Q_{1}+\cdots+Q_{\ell}, I_{n}\right)=\operatorname{rk}\left(Q_{1}+\cdots+Q_{\ell}\right) \leq 3 k .
$$

Proof. Let $P, P^{\prime} \in \mathrm{gl}_{n}$ be matrices such that $\operatorname{rk}(P), \operatorname{rk}\left(P^{\prime}\right) \leq n / 2-1$. We start with three claims.

(0) For all $Q \sim P$ and $Q^{\prime} \sim P^{\prime}$, we have $\operatorname{rk}\left(Q+Q^{\prime}\right) \geq\left|\operatorname{rk}(P)-\operatorname{rk}\left(P^{\prime}\right)\right|$.

(1) There exist $Q \sim P$ and $Q^{\prime} \sim P^{\prime}$ with $\operatorname{rk}\left(Q+Q^{\prime}\right)=\operatorname{rk}(P)+\operatorname{rk}\left(P^{\prime}\right)$.

(2) There exist $Q \sim P$ and $Q^{\prime} \sim P^{\prime}$ with $\operatorname{rk}\left(Q+Q^{\prime}\right) \leq \max \left(\operatorname{rk}(P), \operatorname{rk}\left(P^{\prime}\right)\right)$. 
Claim (0) is obvious. For (1) and (2), take $m=\max \left(\operatorname{rk}(P), \operatorname{rk}\left(P^{\prime}\right)\right)$ and note that

$$
P \sim\left(\begin{array}{cc}
T R & T R S \\
R & R S
\end{array}\right) \sim\left(\begin{array}{cc}
I_{m} & -S \\
& I_{n-m}
\end{array}\right)^{-1}\left(\begin{array}{cc}
T R & T R S \\
R & R S
\end{array}\right)\left(\begin{array}{cc}
I_{m} & -S \\
I_{n-m}
\end{array}\right)=\left(\begin{array}{cc}
(S+T) R & 0 \\
R & 0
\end{array}\right)
$$

for some matrices $R, S, T$ with $R$ an $(n-m) \times m$ matrix of $\operatorname{rank} \operatorname{rk}(P)$ by Proposition 16 , because otherwise $\operatorname{rk}\left(P, I_{n}\right)<\operatorname{rk}(P)$ would hold. Similarly, we have

$$
P^{\prime} \sim\left(\begin{array}{cc}
\bullet & 0 \\
R^{\prime} & 0
\end{array}\right) \sim\left(\begin{array}{cc}
\bullet & R^{\prime \prime} \\
0 & 0
\end{array}\right)
$$

for some $(n-m) \times m$ matrix $R^{\prime}$ and $m \times(n-m)$ matrix $R^{\prime \prime}$ that both have $\operatorname{rank} \operatorname{rk}\left(P^{\prime}\right)$. Now (1) follows from the fact that

$$
\left(\begin{array}{cc}
\bullet & 0 \\
R & 0
\end{array}\right)+\left(\begin{array}{cc}
\bullet & R^{\prime \prime} \\
0 & 0
\end{array}\right)
$$

has rank $\operatorname{rk}(P)+\operatorname{rk}\left(P^{\prime}\right)$ and (2) follows from the fact that

$$
\left(\begin{array}{ll}
\bullet & 0 \\
R & 0
\end{array}\right)+\left(\begin{array}{cc}
\bullet & 0 \\
R^{\prime} & 0
\end{array}\right)
$$

has rank at most $m$.

Note that, since $6 k \leq n$, if $Q \in \mathfrak{g l}_{n}$ is a matrix with $\operatorname{rk}(Q) \leq 3 k$, then $\operatorname{rk}\left(Q, I_{n}\right)$ equals $\operatorname{rk}(Q)$ as the eigenvalue 0 must have the highest geometric multiplicity. So to prove the lemma it suffices to prove that

$$
k<\operatorname{rk}\left(Q_{1}+\cdots+Q_{\ell}\right) \leq 3 k
$$

for some $Q_{1} \sim P_{1}, \ldots, Q_{\ell} \sim P_{\ell}$ using induction on $\ell$. For $\ell=2$ this follows from (1). Now suppose that $\ell>2$ and

$$
k<\operatorname{rk}\left(Q_{1}+\cdots+Q_{\ell-1}\right) \leq 3 k
$$

for some $Q_{1} \sim P_{1}, \ldots, Q_{\ell-1} \sim P_{\ell-1}$. Using (1) if $\operatorname{rk}\left(Q_{1}+\cdots+Q_{\ell-1}\right) \leq 2 k$ and using (0) and (2) otherwise, we see that

$$
\left.k<\operatorname{rk}\left(g\left(Q_{1}+\cdots+Q_{\ell-1}\right) g^{-1}+Q_{\ell}\right)\right) \leq 3 k
$$

for some $g \in \mathrm{GL}_{n}$ and $Q_{\ell} \sim P_{\ell}$. Since $g Q_{1} g^{-1} \sim P_{1}, \ldots, g Q_{\ell-1} g^{-1} \sim P_{\ell-1}$ and $Q_{\ell} \sim P_{\ell}$ this proves the lemma.

Let $X$ be a $G$-stable closed subset of $V$ and let $X_{i}$ be the closure of the projection of $X$ to $\mathfrak{g l}_{n_{i}} / \operatorname{span}\left(I_{n_{i}}\right)$.

Lemma 30. Suppose that $l_{i}+r_{i} \geq 2$ for all $i \in \mathbb{N}$. If there exists a $k \in \mathbb{Z}_{\geq 0}$ such that $X_{i}$ only contains elements $P$ mod $I_{n_{i}}$ with $\operatorname{rk}\left(P, I_{n_{i}}\right) \leq k$ for all $i \gg 0$, then $X \subseteq\{0\}$.

Proof. The lemma follows by induction on $k$ from the following statement.

$\left.{ }^{*}\right)$ Let $k, i \in \mathbb{N}$ be integers such that $n_{i} \geq 6 k$. If $X_{i+1}$ contains an element $P \bmod I_{n_{i+1}}$ with $\operatorname{rk}\left(P, I_{n_{i+1}}\right)=k$, then $X_{i}$ contains an element $Q \bmod I_{n_{i}}$ with $\operatorname{rk}\left(Q, I_{n_{i}}\right)>k$. 
Let $k, i \in \mathbb{N}$ be integers such that $n_{i} \geq 6 k$ and let $P \bmod I_{n_{i+1}}$ be an element of $X_{i+1}$ with $\operatorname{rk}\left(P, I_{n_{i+1}}\right)=k$. By replacing the representative of the element $P \bmod I_{n_{i+1}}$, we may assume that $\operatorname{rk}(P)=k$. By Lemma 28, we have

$$
g P g^{-1}=\left(\begin{array}{ccccccc}
P_{11} & \ldots & P_{1 l_{i}} & \bullet & \ldots & \bullet & \bullet \\
\vdots & & \vdots & \vdots & & \vdots & \vdots \\
P_{l_{1} 1} & \ldots & P_{l_{i} l_{i}} & \bullet & \ldots & \bullet & \bullet \\
\bullet & \ldots & \bullet & Q_{11} & \ldots & Q_{1 r_{i}} & \bullet \\
\vdots & & \vdots & \vdots & & \vdots & \vdots \\
\bullet & \ldots & \bullet & Q_{r_{i} 1} & \ldots & Q_{r_{i} r_{i}} & \bullet \\
\bullet & \ldots & \bullet & \bullet & \ldots & \bullet & \bullet
\end{array}\right)
$$

for $P_{11}, \ldots, P_{l_{i}, l_{i}}, Q_{11}, \ldots, Q_{r_{i} r_{i}} \in \mathrm{gl}_{n_{i}}$ with $\operatorname{rk}\left(P_{11}\right)=k$ for some matrix $g \in \mathrm{GL}_{n_{i+1}}$. Since this is an open condition on $g$, the matrix $\mathrm{gPg}^{-1}$ is in fact of this form for sufficiently general $g \in \mathrm{GL}_{n_{i+1}}$. This allows us to assume that $\operatorname{rk}\left(P_{j j}\right)=k$ for all $j \in\left[l_{i}\right]$ and $\operatorname{rk}\left(-Q_{\ell \ell}^{T}\right)=\operatorname{rk}\left(Q_{\ell \ell}\right)=k$ for all $\ell \in\left[r_{i}\right]$. Lemma 29 now tell us that by replacing $g$ by $\operatorname{Diag}\left(g_{1}, \ldots, g_{l_{i}+r_{i}}, I_{z_{i}}\right) g$ for some $g_{1}, \ldots, g_{l_{i}+r_{i}} \in \mathrm{GL}_{n_{i}}$, we may also assume that

$$
Q=\sum_{j=1}^{l_{i}} P_{j j}-\sum_{\ell=1}^{r_{i}} Q_{\ell \ell}^{T}
$$

satisfies $k<\operatorname{rk}\left(Q, I_{n_{i}}\right)$ and this proves $\left(^{*}\right)$.

Let $n \in \mathbb{N}$ be a multiple of $\operatorname{char}(K)$. Then the trace function on $\mathfrak{g l}_{n}$ is an element of $K\left[\mathfrak{g l}_{n} / \operatorname{span}\left(I_{n}\right)\right]^{\mathrm{SL}_{n}}$. Note that if $\operatorname{char}(K) \mid n_{i}$ and $z_{i}=0$, then $\operatorname{char}(K) \mid n_{i+1}$. So if in addition $\operatorname{char}(K)=2$ or $r_{i}=0$, then the map

$$
\left(\begin{array}{cccccc}
P_{11} & \ldots & P_{1 l_{i}} & \bullet & \ldots & \bullet \\
\vdots & & \vdots & \vdots & & \vdots \\
P_{l_{i} 1} & \ldots & P_{l_{i} l_{i}} & \bullet & \ldots & \bullet \\
\bullet & \ldots & \bullet & Q_{11} & \ldots & Q_{1 r_{i}} \\
\vdots & & \vdots & \vdots & & \vdots \\
\bullet & \ldots & \bullet & Q_{r_{i} 1} & \ldots & Q_{r_{i} r_{i}}
\end{array}\right) \bmod I_{n_{i+1}} \mapsto \sum_{k=1}^{l_{i}} P_{k k}-\sum_{\ell=1}^{r_{i}} Q_{\ell \ell}^{T} \bmod I_{n_{i}} .
$$

commutes with taking the trace.

Definition 31. When $\operatorname{char}(K) \mid n_{i}$ and $z_{i}=0$ for all $i \gg 0$ and in addition $\operatorname{char}(K)=2$ or $r_{i}=0$ for all $i \gg 0$, define the trace of an element $\left(P_{i} \bmod I_{n_{i}}\right)_{i} \in V$ to be the $\mu \in K$ such that $\operatorname{tr}\left(P_{i}\right)=\mu$ for all $i \gg 0$. Otherwise, define the trace of any element of $V$ to be zero.

Note that in all cases the trace of an element of $V$ is $G$-invariant. For $\mu \in K$, denote the $G$-stable closed subset $\{P \in V \mid \operatorname{tr}(P)=\mu\}$ of $V$ by $Y_{\mu}$. Denote the closure of the projection of $Y_{\mu}$ to $\mathrm{gl}_{n_{i}} / \operatorname{span}\left(I_{n_{i}}\right)$ by $Y_{\mu, i}$.

Theorem 32. Assume that $l_{i}+r_{i} \geq 2$ for all $i \in \mathbb{N}$ and that $X \subsetneq Y_{\mu}$ for some $\mu \in K$. Suppose that for all $i \in \mathbb{N}$ such that $I\left(Y_{\mu, i}\right) \subsetneq I\left(X_{i}\right)$ and for all non-zero polynomials $f \in I\left(X_{i}\right) \backslash I\left(Y_{\mu, i}\right)$ of minimal degree, the span of the $\mathrm{SL}_{n_{i+1}}$-orbit of the polynomial

$$
f\left(P_{11}+\cdots+P_{l_{i} l_{i}}-Q_{11}^{T}-\cdots-Q_{r_{i} r_{i}}^{T}\right) \in I\left(X_{i+1}\right)
$$


contains a non-zero off-diagonal polynomial. Then either $X=\emptyset$ or $X=\{0\}$.

Proof. Since $X$ is strictly contained in $Y_{\mu}$, there exists an integer $j \geq 2$ such that $I\left(Y_{\mu, j}\right) \subsetneq I\left(X_{j}\right)$. Note that $I\left(Y_{\mu, i}\right) \subsetneq I\left(X_{i}\right)$ for all integers $i \geq j$. For all $i \geq j$, let $f_{i} \in I\left(X_{i}\right) \backslash I\left(Y_{\mu, i}\right)$ be an element of minimal degree $d_{i}$. Then $d_{i} \leq d_{j}$ for all $i \geq j$ and by choosing $j$ large enough we may assume that $d_{j} \leq n_{j}$.

For $i \geq j$, let $g_{i} \in I\left(X_{i+1}\right)$ be a non-zero off-diagonal polynomial contained in the span of the $\mathrm{SL}_{n_{i+1}}$-orbit of $f_{i}\left(P_{11}+\cdots+P_{l_{i} l_{i}}-Q_{11}^{T}-\cdots-Q_{r_{i} r_{i}}^{T}\right)$. Then $\operatorname{deg}(g) \leq d_{i} \leq$ $d_{j} \leq n_{j} \leq n_{i+1} / 2$ since $n_{i+1}=\left(l_{i}+r_{i}\right) n_{i}+z_{i} \geq 2 n_{i}$. So by Remark 19 and Lemma 30 , we see that $X \subseteq\{0\}$.

Corollary 33. Assume that $l_{i}+r_{i} \geq 2$ for all $i \in \mathbb{N}$. Suppose that for all $\mu \in K$, for all G-stable closed subsets $X \subsetneq Y_{\mu}$, for all $i \in \mathbb{N}$ such that $I\left(Y_{\mu, i}\right) \subsetneq I\left(X_{i}\right)$ and for all non-zero polynomials $f \in I\left(X_{i}\right) \backslash I\left(Y_{\mu, i}\right)$ of minimal degree, the span of the $\mathrm{SL}_{n_{i+1}}$ orbit of the polynomial

$$
f\left(P_{11}+\cdots+P_{l_{i} l_{i}}-Q_{11}^{T}-\cdots-Q_{r_{i} r_{i}}^{T}\right) \in I\left(X_{i+1}\right)
$$

contains a non-zero off-diagonal polynomial. Then the irreducible G-stable closed subsets of $V$ are the non-empty subsets among $\{0\}, V$ and $\{v \in V \mid \operatorname{tr}(v)=\mu\}$ for $\mu \in K$ and every $G$-stable closed subset of $V$ is a finite union of irreducible G-stable closed subsets.

Proof. Using Proposition 14, it is easy to check that the mentioned subsets are either irreducible or empty. If the trace map on $V$ is zero, this is just Theorem 32 applied to $\mu=0$. Assume the trace map is non-zero. Then the linear map

$$
\begin{aligned}
\varphi: K & \rightarrow V \\
\mu & \mapsto\left((\mu+1) E_{11}-E_{22} \bmod I_{n_{i}}\right)_{i} .
\end{aligned}
$$

has the property that $\operatorname{tr}(\varphi(\mu))=\mu$ for all $\mu \in K$. Let $X$ be a $G$-stable closed subset of $V$. Then

$$
\varphi^{-1}(X)=\left\{\mu \in K \mid Y_{\mu} \subseteq X\right\}
$$

is a closed subset of $K$. So either $\varphi^{-1}(X)$ is finite or $\varphi^{-1}(X)=K$. By Theorem 32 , the intersection of $X$ with $Y_{0}$ is either $\emptyset,\{0\}$ or $Y_{0}$ and the intersection of $X$ with $Y_{\mu}$ for $\mu \in K \backslash\{0\}$ is either $\emptyset$ or $Y_{\mu}$. So either

$$
X=\{0\} \cup \bigcup_{\mu \in \varphi^{-1}(X) \backslash\{0\}} Y_{\mu}
$$

or

$$
X=\bigcup_{\mu \in \varphi^{-1}(X)} Y_{\mu}
$$

when $\varphi^{-1}(X)$ is finite and $X=V$ when $\varphi^{-1}(X)=K$.

What remains is reduce the cases (2)-(4b) of Theorem 13 to sequences

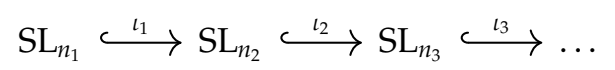

where the conditions of the corollary are satisfied. 
Case (2): $\alpha+\beta=\gamma=\infty$. Since $\gamma=\infty$, we do not have $z_{i}=0$ for all $i \gg 0$. So we get $Y_{0}=V$ and $Y_{\mu}=\emptyset$ for all $\mu \in K \backslash\{0\}$. By restricting to an infinite subsequence we may assume that $l_{i}+r_{i} \geq 2$ and $z_{i} \geq n_{i}$ for all $i \in \mathbb{N}$. Let $i \in \mathbb{N}$ be such that $I\left(X_{i}\right) \neq 0$ and let $f \in I\left(X_{i}\right)$ be a non-zero polynomial of minimal degree. Take $l=l_{i}$, $r=r_{i}, z=z_{i}, m=n_{i}$ and $n=n_{i+1}=(l+r) m+z$. To prove that the conditions of Corollary 33 are satisfied, we need to check the following condition:

$\left.{ }^{*}\right)$ The span of the $\mathrm{SL}_{n}$-orbit of the polynomial

$$
g:=f\left(P_{11}+\cdots+P_{l l}-Q_{11}^{T}-\cdots-Q_{r r}^{T}\right)
$$

contains a non-zero off-diagonal polynomial.

Consider the matrix

$$
H=\left(\begin{array}{ccccccc}
P_{11} & \ldots & P_{1 l} & \bullet & \ldots & \bullet & \bullet \\
\vdots & & \vdots & \vdots & & \vdots & \vdots \\
P_{l 1} & \ldots & P_{l l} & \bullet & \ldots & \bullet & \bullet \\
\bullet & \ldots & \bullet & Q_{11} & \ldots & Q_{1 r} & \bullet \\
\vdots & & \vdots & \vdots & & \vdots & \vdots \\
\bullet & \ldots & \bullet & Q_{r 1} & \ldots & Q_{r r} & \bullet \\
R_{1} & \ldots & R_{l} & \bullet & \ldots & \bullet & \bullet \\
\bullet & \ldots & \bullet & \bullet & \ldots & \bullet & \bullet
\end{array}\right)
$$

where $P_{k, \ell}, Q_{k, \ell}, R_{k} \in \mathfrak{g l}_{m}$. For $\lambda \in K$, consider the matrix

$$
A(\lambda)=\left(\begin{array}{cccccccc}
I_{m} & & & & & & \lambda I_{m} & \\
& \ddots & & & & & & \\
& & I_{m} & & & & & \\
& & & I_{m} & & & & \\
& & & & \ddots & & & \\
& & & & & I_{m} & & \\
& & & & & & I_{m} & \\
& & & & & & & I_{z-m}
\end{array}\right) .
$$

For all $\lambda \in K$, we have

$$
A(\lambda) H A(\lambda)^{-1}=\left(\begin{array}{ccccccc}
P_{11}^{\prime} & \ldots & P_{1 l}^{\prime} & \bullet & \ldots & \bullet & \bullet \\
\vdots & & \vdots & \vdots & & \vdots & \vdots \\
P_{l 1}^{\prime} & \ldots & P_{l l}^{\prime} & \bullet & \ldots & \bullet & \bullet \\
\bullet & \ldots & \bullet & Q_{11} & \ldots & Q_{1 r} & \bullet \\
\vdots & & \vdots & \vdots & & \vdots & \vdots \\
\bullet & \ldots & \bullet & Q_{r 1} & \ldots & Q_{r r} & \bullet \\
\bullet & \ldots & \bullet & \bullet & \ldots & \bullet & \bullet
\end{array}\right)
$$

where $P_{11}^{\prime}=P_{11}+\lambda R_{1}$ and $P_{j j}^{\prime}=P_{j j}$ for all $j \in\{2, \ldots, l\}$. This means that if we let $A(\lambda)$ act on $g$, we obtain the polynomial $h(\lambda)=f\left(P_{11}+\cdots+P_{l l}-Q_{11}^{T}-\cdots-Q_{r r}^{T}+\lambda R_{1}\right)$. Let $d$ be the degree of $f$ and let $f_{d}=f_{d}(P)$ be the homogeneous part of $f$ of degree $d$. Then $f_{d}\left(R_{1}\right)$ is a non-zero off-diagonal polynomial on $\mathrm{gl}_{n}$ since $m \leq(n-1) / 2$. Since $f_{d}\left(R_{1}\right)$ is the coefficient of $h(\lambda)$ at $\lambda^{d}$, it is contained in this span of the $h(\lambda)$. So $\left(^{*}\right)$ holds. So we can apply Corollary 33 and this proves Theorem 13 in case (2). 
Case (3a): $\beta=\infty, \gamma<\infty$ and $\operatorname{char}(K) \neq 2$ or $2 \nmid n_{i}$ for all $i \gg 0$. We do not have $r_{i}=0$ for all $i \gg 0$. Furthermore, if $\operatorname{char}(K)=2$, then $\operatorname{char}(K) \mid n_{i}$ for all $i \gg 0$ does not hold. So we again get $Y_{0}=V$ and $Y_{\mu}=\emptyset$ for all $\mu \in K \backslash\{0\}$. By restricting to an infinite subsequence we may assume that $r_{i}>0, l_{i}+r_{i}>2$ and $z_{i}=0$ for all $i \in \mathbb{N}$. To assume that $l_{i}+r_{i}>2$, we use [BZ, Proposition 2.4]. If $\operatorname{char}(K)=2$, we may furthermore assume that $2 \nmid n_{i}$ for all $i \in \mathbb{N}$. Let $i \in \mathbb{N}$ be such that $I\left(X_{i}\right) \neq 0$ and let $f \in I\left(X_{i}\right)$ be a non-zero polynomial of minimal degree. Take $l=l_{i}, r=r_{i}, m=n_{i}$ and $n=n_{i+1}=(l+r) m$. To prove that the conditions of Corollary 33 are satisfied, we need to check the following condition:

${ }^{*}$ ) The span of the $\mathrm{SL}_{n}$-orbit of the polynomial

$$
g:=f\left(P_{11}+\cdots+P_{l l}-Q_{11}^{T}-\cdots-Q_{r r}^{T}\right)
$$

contains a non-zero off-diagonal polynomial.

Consider the matrix

$$
H=\left(\begin{array}{cccccc}
P_{11} & \ldots & P_{1 l} & \bullet & \ldots & \bullet \\
\vdots & & \vdots & \vdots & & \vdots \\
P_{l 1} & \ldots & P_{l l} & \bullet & \ldots & \bullet \\
R_{11} & \ldots & R_{1 l} & Q_{11} & \ldots & Q_{1 r} \\
\vdots & & \vdots & \vdots & & \vdots \\
R_{r 1} & \ldots & R_{r l} & Q_{r 1} & \ldots & Q_{r r}
\end{array}\right)
$$

where $P_{k, \ell}, Q_{k, \ell}, R_{k} \in \mathfrak{g l}_{m}$. Also consider the matrix

$$
A(\Lambda)=\left(\begin{array}{cccccc}
I_{m} & & & \Lambda & & \\
& \ddots & & & & \\
& & I_{m} & & & \\
& & & I_{m} & & \\
& & & & \ddots & \\
& & & & & I_{m}
\end{array}\right)
$$

for $\Lambda \in \mathfrak{g l}_{m}$. For all $\Lambda \in \mathfrak{g l}_{m}$, we have

$$
A(\Lambda) H A(\Lambda)^{-1}=\left(\begin{array}{cccccc}
P_{11}^{\prime} & \ldots & P_{1 l}^{\prime} & \bullet & \ldots & \bullet \\
\vdots & & \vdots & \vdots & & \vdots \\
P_{l 1}^{\prime} & \ldots & P_{l l}^{\prime} & \bullet & \ldots & \bullet \\
\bullet & \ldots & \bullet & Q_{11}^{\prime} & \ldots & Q_{1 r}^{\prime} \\
\vdots & & \vdots & \vdots & & \vdots \\
\bullet & \ldots & \bullet & Q_{r 1}^{\prime} & \ldots & Q_{r r}^{\prime}
\end{array}\right)
$$

where

$$
\begin{aligned}
P_{11}^{\prime} & =P_{11}+\Lambda R_{11} \\
P_{j j}^{\prime} & =P_{j j} \text { for } j \in\{2, \ldots, l\} \\
Q_{11}^{\prime} & =Q_{11}-R_{11} \Lambda \\
Q_{j j}^{\prime} & =Q_{\ell \ell} \text { for } \ell \in\{2, \ldots, r\} .
\end{aligned}
$$

This means that if we let $A(\Lambda)$ act on the polynomial $g$, we obtain the polynomial $h(\Lambda)=f\left(P_{11}+\cdots+P_{l l}-Q_{11}^{T}-\cdots-Q_{r r}^{T}+\Lambda R_{11}+\Lambda^{T} R_{11}^{T}\right)$. Let $d$ be the degree 
of $f$ and let $f_{d}=f_{d}(P)$ be the homogeneous part of $f$ of degree $d$. Then we see that the homogeneous part of $h(\Lambda)$ of degree $d$ in the coordinates of $\Lambda$ equals $f_{d}\left(\Lambda R_{11}+\Lambda^{T} R_{11}^{T}\right)$.

To prove that $f_{d}\left(\Lambda R_{11}+\Lambda^{T} R_{11}^{T}\right)$ is non-zero as a polynomial in $\Lambda$ and $R_{11}$, we will use reduction rules for graphs. See for example [BA] for more on this. Let $\Gamma$ be an undirected multigraph. Denote its vertex and edge sets by $V(\Gamma)$ and $E(\Gamma)$.

Definition 34. We consider the following three reduction rules:

(1) Remove an edge from $\Gamma$.

(2) Remove a vertex of $\Gamma$ that has at least one loop.

(3) Pick a vertex $v$ of $\Gamma$ that has a least one loop. Replace an edge of $\Gamma$ with endpoints $v \neq w$ by a loop at $w$.

We say that $\Gamma$ reduces to a multigraph $\Gamma^{\prime}$ if $\Gamma^{\prime}$ can be obtained from $\Gamma$ by applying a series of reductions.

Lemma 35. If $\Gamma$ reduces to the empty graph, then the linear map

$$
\begin{aligned}
\ell_{\Gamma}: K^{E(\Gamma)} & \rightarrow K^{V(\Gamma)} \\
\left(x_{e}\right)_{e} & \mapsto\left(\sum_{e \ni v} x_{e}\right)_{v}
\end{aligned}
$$

is surjective. Here entries corresponding to loops are only added once.

Proof. If $\Gamma$ is the empty graph, then $\ell_{\Gamma}$ is surjective. So it suffices to check that $\ell_{\Gamma}$ is surjective whenever we have a reduction $\Gamma^{\prime}$ of $\Gamma$ such that the similarly defined map $\ell_{\Gamma^{\prime}}$ is surjective. When $\Gamma^{\prime}$ is obtained from $\Gamma$ by applying reduction rule (1), this is easy. The other cases follow from the fact that $x_{e}$ only appears in coordinate $v$ when $e$ is a loop with endpoint $v$.

\section{Lemma 36.}

(a) If $\operatorname{char}(K) \neq 2$, then $\left\{P Q+P^{T} Q^{T} \mid P, Q \in \mathfrak{g l}_{n}\right\}=\mathfrak{g l}_{n}$ for all $n \in \mathbb{N}$.

(b) If $\operatorname{char}(K)=2$, then $\left\{P Q+P^{T} Q^{T} \mid P, Q \in \mathfrak{g l}_{n}\right\}$ is dense in $\mathfrak{s l}_{n}$ for all $n \in \mathbb{N}$.

Proof. In part (a) we can even take $P$ and $Q$ to be symmetric, because by [Ta, (ii)] every matrix is a product of two symmetric matrices. For part (b), suppose that $\operatorname{char}(K)=2$ and let $n \in \mathbb{N}$ be an integer. Then $P Q+P^{T} Q^{T} \in \mathfrak{s l}_{n}$ for all $P, Q \in \mathfrak{g l}_{n}$. Note that $\left\{P Q+P^{T} Q^{T} \mid P, Q \in \mathfrak{g l}_{n}\right\}$ is dense in $\mathfrak{s l}_{n}$ if and only if the morphism

$$
\begin{aligned}
\varphi: \mathfrak{g l}_{n} \times \mathfrak{g l}_{n} & \rightarrow \mathrm{gl}_{n} / \operatorname{span}\left(E_{n, n}\right) \\
(P, Q) & \mapsto P Q+P^{T} Q^{T} \bmod E_{n, n}
\end{aligned}
$$

is dominant. To show that $\varphi$ is dominant, it suffices to show that its derivative

$$
\begin{aligned}
\mathrm{d}_{(R, S)} \varphi: \mathfrak{g l}_{n} \oplus \mathfrak{g l}_{n} & \rightarrow \mathfrak{g l}_{n} / \operatorname{span}\left(E_{n, n}\right) \\
(P, Q) & \mapsto P S+P^{T} S^{T}+R Q+R^{T} Q^{T} \bmod E_{n, n}
\end{aligned}
$$

at the point

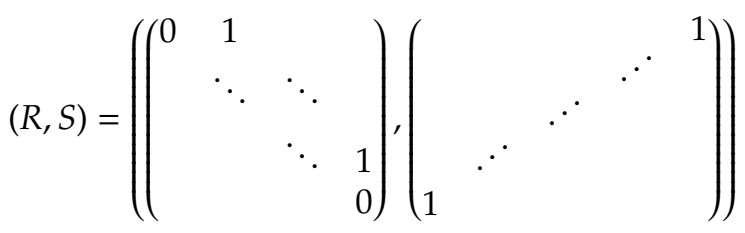


is surjective. Note that

$$
\begin{aligned}
\left(\mathrm{d}_{(R, S)} \varphi\right)\left(E_{i, j}, 0\right) & =E_{i, n+1-j}+E_{j, n+1-i} \\
\left(\mathrm{~d}_{(R, S)} \varphi\right)\left(0, E_{k, \ell}\right) & =\left(1-\delta_{k 1}\right) E_{k-1, \ell}+\left(1-\delta_{\ell n}\right) E_{\ell+1, k}
\end{aligned}
$$

and hence $\left(\mathrm{d}_{(R, S)} \varphi\right)\left(0, E_{1, n}\right)=0$ and $\left(\mathrm{d}_{(R, S)} \varphi\right)\left(E_{i, i}, 0\right)=0$ for all $i \in[n]$, because $\operatorname{char}(K)=2$. The other basis elements of $\mathfrak{g l}_{n} \oplus \mathfrak{g l}_{n}$ all get sent to a sum of one or two basis elements of $\mathrm{gl}_{n} / \operatorname{span}\left(E_{n, n}\right)$. To prove that $\mathrm{d}_{(R, S)} \varphi$ is surjective, it suffices by the previous lemma to prove that the restriction of $d_{(R, S)} \varphi$ to the span of these other basis vectors equals $\ell_{\Gamma}$ for some multigraph $\Gamma$ that reduces to the empty graph.

Define the multigraph $\Gamma$ as follows: We let $V(\Gamma)$ be the basis $\left\{E_{i, j} \mid(i, j) \neq(n, n)\right\}$ of $\mathfrak{g I}_{n} / \operatorname{span}\left(E_{n, n}\right)$ and we let $E(\Gamma)$ be the set

$$
\left\{\left(E_{i, j}, 0\right) \mid i \neq j\right\} \cup\left\{\left(0, E_{k, \ell}\right) \mid k, \ell \in[n]\right\} \backslash\left\{\left(0, E_{1, n}\right)\right\}
$$

of basis element of $\mathfrak{g l}_{n} \oplus \mathfrak{g l}_{n}$ that are not mapped to 0 . This allows to define the set of endpoints of an edge in such a way that $\left.\left(\mathrm{d}_{(R, S)} \varphi\right)\right|_{\operatorname{span}(E(\Gamma))}=\ell_{\Gamma}$. Next we check that $\Gamma$ reduces to the empty graph. One can check that $\Gamma$ has two loops at $E_{1,1}$, a loop at $E_{k, 1}$ for all $k>1$ and a loop at $E_{\ell, n}$ for all $\ell<n$. We also have:

(x) edges with endpoints $E_{i, j}$ and $E_{j+1, i+1}$ for all $i, j \in[n-1]$;

(y) edges with endpoints $E_{k, 1}$ and $E_{n, n+1-k}$ for all $1<k<n$; and

(z) edges with endpoints $E_{\ell, n}$ and $E_{1, n+1-\ell}$ for $1<\ell<n$.

First, we remove all other edges from $\Gamma$ using reduction rule (1). Next, we replace the edges (y) and (z) by loops at $E_{n, k}$ for $1<k<n$ and $E_{1, \ell}$ for $1<\ell<n$ using reduction rule (3). The graph $\Gamma^{\prime}$ obtained this way has has the edges (x) together with loops at $E_{1,1}$ and $E_{1, i}, E_{n, i}, E_{i, 1}, E_{i, n}$ for $1<i<n$. Now consider the connected components of $\Gamma^{\prime}$. One connected component consists of a path from $E_{1,1}$ to $E_{n, n}$ with a loop at $E_{1,1}$. All other components are path with loops at both ends starting at a vertex of the form $E_{1, i}$ or $E_{i, 1}$ and ending at a vertex of the form $E_{n, i}$ or $E_{i, n}$. Each of these components reduces to the empty graph by repeatedly using reduction rules (2) and (3). Therefore $\Gamma^{\prime}$ and $\Gamma$ also reduce to the empty graph. Hence $\mathrm{d}_{(R, S)} \varphi$ is surjective and $\varphi$ is dominant.

Since the polynomial $f$ is non-zero, so is $f_{d}$. By combining the lemma with the fact that $f_{d}\left(P+\lambda I_{m}\right)=f_{d}(P)$ for all $P \in \mathfrak{g l}_{m}$ and $\lambda \in K$, we see that the polynomial $f_{d}\left(\Lambda R_{11}+\Lambda^{T} R_{11}^{T}\right)$ is non-zero. Now view $f_{d}\left(\Lambda R_{11}+\Lambda^{T} R_{11}^{T}\right)$ as a polynomial in $\Lambda$ whose coefficients are polynomials in the entries of $R_{11}$. Any of its non-zero coefficients is a non-zero off-diagonal polynomial on $\mathrm{gl}_{n}$ which is contained in the span of the orbit of $g$. Here we use that $m \leq(n-1) / 2$ since $l+r>2$. So $\left({ }^{*}\right)$ holds. So we can apply Corollary 33 and this proves Theorem 13 in case (3a).

Case (3b): $\beta=\infty, \gamma<\infty, \operatorname{char}(K)=2$ and $2 \mid n_{i}$ for all $i \gg 0$. Note that in this case the trace map on $V$ is non-zero. By restricting to an infinite subsequence we may assume that $r_{i}>0, l_{i}+r_{i}>2, z_{i}=0$ and $2 \mid n_{i}$ for all $i \in \mathbb{N}$. Let $\mu \in K$, suppose that $X \subsetneq Y_{\mu}$ and let $i \in \mathbb{N}$ be such that $I\left(Y_{\mu, i}\right) \subsetneq I\left(X_{i}\right)$. Let $f \in I\left(X_{i}\right) \backslash I\left(Y_{\mu, i}\right)$ be a polynomial of minimal degree. Take $l=l_{i}, r=r_{i}, m=n_{i}$ and $n=n_{i+1}=(l+r) n$. To prove that the conditions of Corollary $\mathbf{3 3}$ are satisfied, we need to check the following condition:

$\left.{ }^{*}\right)$ The span of the $\mathrm{SL}_{n}$-orbit of the polynomial

$$
g:=f\left(P_{11}+\cdots+P_{l l}-Q_{11}^{T}-\cdots-Q_{r r}^{T}\right)
$$


contains a non-zero off-diagonal polynomial.

As in case (3a), we find that all coefficients of $f_{d}\left(\Lambda R_{11}+\Lambda^{T} R_{11}^{T}\right)$ are off-diagonal polynomials on $\mathfrak{g l}_{n}$ which are contained in the span of the orbit of $g$. So it suffices to prove that $f_{d}\left(\Lambda R_{11}+\Lambda^{T} R_{11}^{T}\right)$ is not the zero polynomial.

Suppose that the polynomial $f_{d}\left(\Lambda R_{11}+\Lambda^{T} R_{11}^{T}\right)$ is the zero polynomial. Then $f_{d}(P)=0$ for all $P \in \mathfrak{s l}_{m}$ by Lemma 36(b). So $f_{d}$ is a multiple of the trace function on $\mathrm{gl}_{m}$ and we can write $f_{d}=\operatorname{tr} \cdot h$ for some $h$. But then $f-(\operatorname{tr}-\mu) h \in I\left(X_{i}\right) \backslash I\left(Y_{\mu, i}\right)$. This contradicts the minimality of the degree of $f$. So $f_{d}\left(\Lambda R_{11}+\Lambda^{T} R_{11}^{T}\right)$ can not be the zero polynomial. So $\left.{ }^{*}\right)$ again holds. So we can apply Corollary 33 and this proves Theorem 13 in case (3b).

Case (4a): $\beta+\gamma<\infty$ and $\operatorname{char}(K) \nmid n_{i}$ for all $i \gg 0$. We do not have $\operatorname{char}(K) \nmid n_{i}$ for all $i \gg 0$. So we get $Y_{0}=V$ and $Y_{\mu}=\emptyset$ for all $\mu \in K \backslash\{0\}$. By restricting to an infinite subsequence we may assume that $l_{i}>2, r_{i}=z_{i}=0$ and $\operatorname{char}(K) \nmid n_{i}$ for all $i \in \mathbb{N}$. Let $i \in \mathbb{N}$ be such that $I\left(X_{i}\right) \neq 0$ and let $f \in I\left(X_{i}\right)$ be a non-zero polynomial of minimal degree. Take $l=l_{i}, m=n_{i}$ and $n=n_{i+1}=l m$. Then $m \leq(n-1) / 2$. To prove that the conditions of Corollary 33 are satisfied, we need to check the following condition:

${ }^{*}$ The span of the $\mathrm{SL}_{n}$-orbit of the polynomial

$$
g:=f\left(P_{11}+\cdots+P_{l l}\right)
$$

contains a non-zero off-diagonal polynomial.

Consider the matrix

$$
H=\left(\begin{array}{ccc}
P_{11} & \ldots & P_{1 l} \\
\vdots & & \vdots \\
P_{l 1} & \ldots & P_{l l}
\end{array}\right)
$$

where $P_{k, \ell} \in \mathfrak{g l}_{m}$. Also consider the matrix

$$
A(\Lambda)=\left(\begin{array}{ccccc}
I_{m} & \Lambda & & & \\
& I_{m} & & & \\
& & I_{m} & & \\
& & & \ddots & \\
& & & & I_{m}
\end{array}\right)
$$

for $\Lambda \in \mathfrak{g l}_{m}$. For all $\Lambda \in \mathfrak{g l}_{m}$, we have

$$
A(\Lambda) H A(\Lambda)^{-1}=\left(\begin{array}{ccc}
P_{11}^{\prime} & \ldots & P_{1 l}^{\prime} \\
\vdots & & \vdots \\
P_{l 1}^{\prime} & \ldots & P_{l l}^{\prime}
\end{array}\right)
$$

where $P_{11}^{\prime}=P_{11}+\Lambda P_{21}, P_{22}^{\prime}=P_{22}-P_{21} \Lambda$ and $P_{j j}^{\prime}=P_{j j}$ for $j \in\{3, \ldots, l\}$. This means that if we let $A(\Lambda)$ act on $g$, we obtain the polynomial $h(\Lambda)=f\left(P_{11}+\cdots+P_{l l}+\left[\Lambda, P_{21}\right]\right)$ where $[-,-]$ is the commutator bracket. Let $d$ be the degree of $f$ and let $f_{d}=f_{d}(P)$ be the homogeneous part of $f$ of degree $d$. Then we see that the homogeneous part of $h(\Lambda)$ of degree $d$ in the coordinates of $\Lambda$ equals $f_{d}\left(\left[\Lambda, P_{21}\right]\right)$. Since $f$ is non-zero, so is $f_{d}$. By [St, Theorem 6.3], we know that every element of $\mathrm{gl}_{m}$ is of the form $[X, Y]+\lambda I_{m}$ for some $X, Y \in \mathfrak{g l}_{m}$ and $\lambda \in K$. So since $f_{d}\left(P+\lambda I_{m}\right)=f_{d}(P)$ for all 
$P \in \mathfrak{g l}_{m}$ and $\lambda \in K$, we see that $f_{d}\left(\left[\Lambda, P_{21}\right]\right)$ is not the zero polynomial. Any nonzero coefficient of $f_{d}\left(\left[\Lambda, P_{21}\right]\right)$ as a polynomial in $\Lambda$ satisfies $\left.{ }^{*}\right)$. So we can apply Corollary 33 and this proves Theorem 13 in case (4a).

Case (4b): $\beta+\gamma<\infty$ and $\operatorname{char}(K) \mid n_{i}$ for all $i \gg 0$. Note that in this case the trace map on $V$ is non-zero. By restricting to an infinite subsequence we may assume that $l_{i}>2, r_{i}=z_{i}=0$ and $\operatorname{char}(K) \mid n_{i}$ for all $i \in \mathbb{N}$. We now proceed as in the case (4a) with the same modifications that were established in case (3b).

\section{Limits of Classical groups OF TYPE C}

From now on, we assume that $\operatorname{char}(K) \neq 2$. In this section, we let $G$ be the $\operatorname{direct}$ limit of a sequence

$$
\mathrm{Sp}_{2 n_{1}} \stackrel{\iota_{1}}{\longleftrightarrow} \mathrm{Sp}_{2 n_{2}} \stackrel{\iota_{2}}{\longleftrightarrow} \mathrm{Sp}_{2 n_{3}} \stackrel{\iota_{3}}{\longleftrightarrow \ldots}
$$

of diagonal embeddings given by

$$
\begin{aligned}
\iota_{i}: \mathrm{Sp}_{2 n_{i}} & \hookrightarrow \operatorname{Sp}_{2 n_{i+1}} \\
\left(\begin{array}{cc}
A & B \\
C & D
\end{array}\right) & \mapsto\left(\begin{array}{cc}
\operatorname{Diag}\left(A, \ldots, A, I_{z_{i}}\right) & \operatorname{Diag}(B, \ldots, B, 0) \\
\operatorname{Diag}(C, \ldots, C, 0) & \operatorname{Diag}\left(D, \ldots, D, I_{z_{i}}\right)
\end{array}\right)
\end{aligned}
$$

with $l_{i}$ blocks $A, B, C, D \in \mathfrak{g l}_{n_{i}}$ for some $l_{i} \in \mathbb{N}$ and $z_{i} \in \mathbb{Z}_{\geq 0}$. We let $V$ be the inverse limit of the sequence

$$
\mathfrak{s p}_{2 n_{1}} \longleftarrow \mathfrak{s p}_{2 n_{2}} \longleftarrow \mathfrak{s p}_{2 n_{3}} \longleftarrow
$$

where the maps are given by

$$
\left(\begin{array}{ccccccccc}
P_{11} & \ldots & P_{1 l_{i}} & \bullet & Q_{11} & \ldots & Q_{1 l_{i}} & \bullet \\
\vdots & & \vdots & \vdots & \vdots & & \vdots & \vdots \\
P_{l_{i} 1} & \ldots & P_{l_{i} l_{i}} & \vdots & Q_{l_{i} 1} & \ldots & Q_{l_{i} l_{i}} & \vdots \\
\bullet & \ldots & \ldots & \bullet & \bullet & \ldots & \ldots & \bullet \\
R_{11} & \ldots & R_{1 l_{i}} & \bullet & S_{11} & \ldots & S_{1 l_{i}} & \bullet \\
\vdots & & \vdots & \vdots & \vdots & & \vdots & \vdots \\
R_{l_{i} 1} & \ldots & R_{l_{i} l_{i}} & \vdots & S_{l_{i} 1} & \ldots & S_{l_{i} l_{i}} & \vdots \\
\bullet & \ldots & \ldots & \bullet & \bullet & \ldots & \ldots & \bullet
\end{array}\right) \mapsto\left(\begin{array}{clll}
\sum_{k=1}^{l_{i}} P_{k k} & \sum_{k=1}^{l_{i}} Q_{k k} \\
\sum_{k=1}^{l_{i}} R_{k k} & \sum_{k=1}^{l_{i}} S_{k k}
\end{array}\right)
$$

with $P_{k \ell}=-S_{\ell k^{\prime}}^{T} Q_{k \ell}, R_{k \ell} \in \mathfrak{g l}_{n_{i}}$ such that $Q_{k \ell}=Q_{\ell k}^{T}$ and $R_{k \ell}=R_{\ell k}^{T}$.

Theorem 37. The space $V$ is G-Noetherian.

Let $X \subsetneq V$ be a $G$-stable closed subset. Let $X_{i}$ be the closure of the projection of $X$ to $\mathfrak{s p}_{2 n_{i}}$ and let $I\left(X_{i}\right) \subseteq K\left[\mathfrak{s p}_{2 n_{i}}\right]$ be the ideal of $X_{i}$. If $\#\left\{i \mid l_{i}>1\right\}<\infty$, then Theorem 37ffollows from [ES, Theorem 1.2].

Remark 38. Let $X \subsetneq V$ be a $G$-stable closed subset in the case where $\#\left\{i \mid l_{i}>1\right\}<\infty$. Then $V$ can be identified with a subspace of the space of $\mathbb{N} \times \mathbb{N}$ matrices and we can prove (using technique similar to the ones used in this paper) that $X$ consists of matrices of bounded rank. The G-Noetherianity of $V$ then follows from the $\operatorname{Sym}(\mathbb{N})$-Noetherianity of $K^{\mathbb{N} \times k}$ for $k \in \mathbb{N}$. Important to note here is that, for every 
$n \in \mathbb{N}$, the group $\mathrm{Sp}_{2 n}$ contains all matrices corresponding to permutations $\pi \in S_{2 n}$ such that $\pi(i+n)=\pi(i)+n$ for all $i \in[n]$. This allows us to define an action of $\operatorname{Sym}(\mathbb{N})$ on $V$, up to which the closed subset $X$ is Noetherian. Similar statements hold for sequences of types $B$ and $D$.

We assume that $\#\left\{i \mid l_{i}>1\right\}=\infty$. By restricting to an infinite subsequence, we may assume that $l_{i} \geq 3$ for all $i \in \mathbb{N}$.

Lemma 39. Let $n \in \mathbb{N}$, let $Y \subsetneq \mathfrak{s p}_{2 n}$ be an $\mathrm{Sp}_{2 n}$-stable closed subset and let $\mathrm{Z}$ be the closed subset

$$
\left\{\left(\begin{array}{cc}
P & Q \\
R & -P^{T}
\end{array}\right) \in \mathfrak{s p}_{2 n} \mid P=P^{T}\right\}
$$

of $\mathfrak{s p}_{2 n}$. Then there is a non-zero polynomial $f \in I(Y)$ whose top-graded part is not contained in the ideal of $Z$.

Proof. Since $Y \subsetneq \mathfrak{s p}_{2 n}$, there is a non-zero polynomial $f \in I(Y)$. Since $f$ is nonzero, so is its top-graded part $g$. Let the group $\mathrm{GL}_{n}$ act on $\mathfrak{s p}_{2 n}$ via the diagonal embedding $\mathrm{GL}_{n} \hookrightarrow \mathrm{Sp}_{2 n^{\prime}}, A \mapsto \operatorname{Diag}\left(A, A^{-T}\right)$. Then we get a action of $\mathrm{GL}_{n}$ on $K\left[\mathfrak{s p}_{2 n}\right]$. Note that this action respects the grading on $K\left[\mathfrak{s p}_{2 n}\right]$ and that the ideal $I(Y)$ is $\mathrm{GL}_{n}$-stable. So for all $A \in \mathrm{GL}_{n}$ we have $A \cdot f \in I(Y)$ and the top-graded part of this polynomial is $A \cdot g$. Hence it suffices to prove that $A \cdot g \notin I(Z)$ for some $A \in \mathrm{GL}_{n}$. Note that

$$
\begin{aligned}
\mathrm{GL}_{n} \cdot Z & =\left\{A \cdot\left(\begin{array}{cc}
P & Q \\
R & -P^{T}
\end{array}\right) \mid \begin{array}{c}
P=P^{T}, A \in \mathrm{GL}_{n} \\
Q=Q^{T}, R=R^{T}
\end{array}\right\} \\
& =\left\{\left(\begin{array}{cc}
A P A^{-1} & A Q A^{T} \\
A^{-T} R A^{-1} & -A^{-T} P^{T} A^{T}
\end{array}\right) \mid \begin{array}{c}
P=P^{T}, A \in \mathrm{GL}_{n} \\
Q=Q^{T}, R=R^{T}
\end{array}\right\} \\
& =\left\{\left(\begin{array}{cc}
A P A^{-1} & Q \\
R & -\left(A P A^{-1}\right)^{T}
\end{array}\right) \mid \begin{array}{l}
P=P^{T}, A \in \mathrm{GL}_{n} \\
Q=Q^{T}, R=R^{T}
\end{array}\right\}
\end{aligned}
$$

and that $\left\{A P A^{-1} \mid P=P^{T}, A \in \mathrm{GL}_{n}\right\}$ is dense in $\mathrm{gl}_{n}$ since $K$ is infinite and diagonal matrices are symmetric. So $\mathrm{GL}_{n} \cdot \mathrm{Z}$ is dense in $\mathfrak{s p}_{2 n}$. So since the polynomial $g$ is non-zero, there must be an $A \in \mathrm{GL}_{n}$ such that $A \cdot g \notin I(Z)$.

Lemma 40. Let $i \in \mathbb{N}$ and let $f=f(P, Q, R) \in I\left(X_{i}\right)$ be a non-zero polynomial whose top-graded part $g$ is not contained in the ideal of

$$
\left\{\left(\begin{array}{cc}
P & Q \\
R & -P^{T}
\end{array}\right) \in \mathfrak{s p}_{2 n_{i}} \mid P=P^{T}\right\} .
$$

Then $I\left(X_{i+1}\right) \cap K\left[r_{k \ell} \mid 1 \leq k, \ell \leq n_{i+1}\right] /\left(r_{k \ell}-r_{\ell k}\right)$ contains a non-zero polynomial with degree at most $\operatorname{deg}(f)$. 
Proof. Take $m=n_{i}, l=l_{i}, z=z_{i}$ and $n=n_{i+1}=l m+z$. Consider the matrix

$$
H=\left(\begin{array}{cccccccc}
P_{11} & \ldots & P_{1 l} & \bullet & Q_{11} & \ldots & Q_{1 l} & \bullet \\
\vdots & & \vdots & \vdots & \vdots & & \vdots & \vdots \\
P_{l 1} & \ldots & P_{l l} & \vdots & Q_{l 1} & \ldots & Q_{l l} & \vdots \\
\bullet & \ldots & \ldots & \bullet & \bullet & \ldots & \ldots & \bullet \\
R_{11} & \ldots & R_{1 l} & \bullet & S_{11} & \ldots & S_{1 l} & \bullet \\
\vdots & & \vdots & \vdots & \vdots & & \vdots & \vdots \\
R_{l 1} & \ldots & R_{l l} & \vdots & S_{l 1} & \ldots & S_{l l} & \vdots \\
\bullet & \ldots & \ldots & \bullet & \bullet & \ldots & \ldots & \bullet
\end{array}\right) \in \mathfrak{p}_{2 n}
$$

and consider the matrix

$$
A(\lambda)=\left(\begin{array}{cccccc}
I_{m} & & & & \lambda I_{m} & \\
& I_{m} & & \lambda I_{m} & & \\
& & I_{n-2 m} & & & \\
& & & I_{m} & & \\
& & & & I_{m} & \\
& & & & & I_{n-2 m}
\end{array}\right) \in \mathrm{Sp}_{2 n}
$$

for $\lambda \in K$. The polynomial $f=f(P, Q, R) \in I\left(X_{i}\right)$ pulls back to the element

$$
f\left(\sum_{k=1}^{l} P_{k k}, \sum_{k=1}^{l} Q_{k k}, \sum_{k=1}^{l} R_{k k}\right)
$$

of $I\left(X_{i+1}\right)$. For $\lambda \in K$, we have

$$
A(\lambda) H A(\lambda)^{-1}=\left(\begin{array}{cccccccc}
P_{11}^{\prime} & \ldots & P_{1 l}^{\prime} & \bullet & Q_{11}^{\prime} & \ldots & Q_{1 l}^{\prime} & \bullet \\
\vdots & & \vdots & \vdots & \vdots & & \vdots & \vdots \\
P_{l 1}^{\prime} & \ldots & P_{l l}^{\prime} & \vdots & Q_{l 1}^{\prime} & \ldots & Q_{l l}^{\prime} & \vdots \\
\bullet & \ldots & \ldots & \bullet & \bullet & \ldots & \ldots & \bullet \\
R_{11} & \ldots & R_{1 l} & \bullet & S_{11}^{\prime} & \ldots & S_{1 l}^{\prime} & \bullet \\
\vdots & & \vdots & \vdots & \vdots & & \vdots & \vdots \\
R_{l 1} & \ldots & R_{l l} & \vdots & S_{l 1}^{\prime} & \ldots & S_{l l}^{\prime} & \vdots \\
\bullet & \ldots & \ldots & \bullet & \bullet & \ldots & \ldots & \bullet
\end{array}\right)
$$

where

$$
\begin{aligned}
P_{11}^{\prime} & =P_{11}+\lambda R_{21} \\
P_{22}^{\prime} & =P_{22}+\lambda R_{12} \\
P_{k k}^{\prime} & =P_{k k} \text { for } k=3, \ldots, l \\
Q_{11}^{\prime} & =Q_{11}+\lambda\left(S_{21}-P_{12}\right)-\lambda^{2} R_{22} \\
Q_{22}^{\prime} & =Q_{22}+\lambda\left(S_{12}-P_{21}\right)-\lambda^{2} R_{11} \\
Q_{k k}^{\prime} & =Q_{k k} \text { for } k=3, \ldots, l
\end{aligned}
$$


Let $g$ be the top-graded part of $f$. Then we see that $g\left(R_{21}+R_{12},-\left(R_{11}+R_{22}\right), \sum_{k=1}^{l} R_{k k}\right)$ is contained in the span of

$$
A(\lambda) \cdot f\left(\sum_{k=1}^{l} P_{k k}, \sum_{k=1}^{l} Q_{k k}, \sum_{k=1}^{l} R_{k k}\right)
$$

over all $\lambda \in K$. We have $g(P, Q, R) \neq 0$ for some symmetric matrices $P, Q, R \in \mathfrak{g l}_{m}$. Since $\operatorname{char}(K) \neq 2$, there are matrices $R_{12}, R_{21}$ such that $R_{12}=R_{21}^{T}$ and $R_{21}+R_{12}=P$. And, since $l>2$, there are symmetric matrices $R_{11}, \ldots, R_{l l}$ such that $-\left(R_{11}+R_{22}\right)=Q$ and $\sum_{k=1}^{l} R_{k k}=R$. So we see that the polynomial

$$
g\left(R_{21}+R_{12},-\left(R_{11}+R_{22}\right), \sum_{k=1}^{l} R_{k k}\right) \in I\left(X_{i+1}\right)
$$

is non-zero.

Since $X \subsetneq V$, we know that $X_{j} \subsetneq \mathfrak{s p}_{2 n_{j}}$ for some $j \in \mathbb{N}$. Using the previous lemma, we see that there is a $d \in \mathbb{Z}_{\geq 0}$ such that $I\left(X_{i}\right) \cap K\left[r_{k \ell} \mid 1 \leq k, \ell \leq n_{i}\right] /\left(r_{k \ell}-r_{\ell k}\right)$ contains a non-zero polynomial of degree at most $d$ for all $i>j$.

Lemma 41. Let $n \in \mathbb{N}$, let $Y \subsetneq \mathfrak{s p}_{2 n}$ be an $\mathrm{Sp}_{2 n}$-stable closed subset, let

$$
M=\left(\begin{array}{ll}
M_{11} & M_{12} \\
M_{21} & M_{22}
\end{array}\right) \in Y
$$

be an element and suppose that

$$
I(Y) \cap K\left[r_{k \ell} \mid 1 \leq k, \ell \leq n\right] /\left(r_{k \ell}-r_{\ell k}\right)
$$

contains a non-zero polynomial of degree $m+1$. Then $\operatorname{rk}\left(M_{12}\right), \operatorname{rk}\left(M_{21}\right) \leq m$. Furthermore, if $n>6 m$, then $\operatorname{rk}\left(M_{11}\right)=\operatorname{rk}\left(M_{22}\right) \leq 3 m / 2$ and $\operatorname{rk}(M) \leq 5 m$.

Proof. Let $\mathrm{GL}_{n}$ act on $\mathfrak{s p}_{2 n}$ via the diagonal embedding

$$
\begin{aligned}
\mathrm{GL}_{n} & \hookrightarrow \mathrm{Sp}_{2 n} \\
g & \mapsto \operatorname{Diag}\left(g, g^{-T}\right)
\end{aligned}
$$

and on $\left\{R \in \mathfrak{g l}_{n} \mid R=R^{T}\right\}$ by $g \cdot R=g^{-T} R g^{-1}$. Then the projection map

$$
\begin{aligned}
& \pi: \mathfrak{s p}_{2 n} \rightarrow \mathrm{gl}_{n} \\
& \left(\begin{array}{ll}
P & Q \\
R & S
\end{array}\right) \mapsto R
\end{aligned}
$$

is $\mathrm{GL}_{n}$-equivairant. Let $Z$ be the closure of $\pi(Y)$ in $\left\{R \in \mathfrak{g l}_{n} \mid R=R^{T}\right\}$. Since $Y$ is $\mathrm{GL}_{n}$-stable, so are $\pi(Y)$ and $Z$. Since $\operatorname{char}(K) \neq 2$, the $\mathrm{GL}_{n}$-orbits of $\left\{R \in \mathrm{gl}_{n} \mid R=R^{T}\right\}$ consist of all symmetric matrices of equal rank. So $Z$ must consist of all symmetric matrices of rank at most $h$ for some $h \leq n$. Since $I(Z)$ contains a non-zero polynomial of degree $m+1$, we see that $h \leq m$. See, for example, [SS, §4]. So

$$
Y \subseteq\left\{\left(\begin{array}{ll}
P & Q \\
R & S
\end{array}\right) \in \mathfrak{s p}_{2 n} \mid \operatorname{rk}(R) \leq m\right\} .
$$

Let $A \in \mathfrak{g l}_{n}$ be a symmetric matrix. Then we have

$$
\left(\begin{array}{cc}
0 & I_{n} \\
-I_{n} & A
\end{array}\right) \in \mathrm{Sp}_{2 n}
$$


with inverse

$$
\left(\begin{array}{cc}
A & -I_{n} \\
I_{n} & 0
\end{array}\right)
$$

Let

$$
\left(\begin{array}{ll}
P & Q \\
R & S
\end{array}\right)
$$

be an element of $Y$. Then

$$
\left(\begin{array}{cc}
0 & I_{n} \\
-I_{n} & A
\end{array}\right)\left(\begin{array}{ll}
P & Q \\
R & S
\end{array}\right)\left(\begin{array}{cc}
0 & I_{n} \\
-I_{n} & A
\end{array}\right)^{-1}=\left(\begin{array}{cc}
\bullet & \bullet \\
A R A+A S-P A-Q & \bullet
\end{array}\right) \in Y
$$

So we get $\operatorname{rk}(A R A+A S-P A-Q) \leq m$ for all symmetric matrices $A \in \mathfrak{g l}_{n}$. For $A=0$, this gives us $\operatorname{rk}(Q) \leq m$ and so $\operatorname{rk}\left(M_{12}\right) \leq m$ in particular. For all $A$, we can write

$$
P A+(P A)^{T}=(A R A+A S-P A-Q)-A R A+Q
$$

since $S=-P^{T}$. We get

$$
\operatorname{rk}\left(P A+(P A)^{T}\right) \leq \operatorname{rk}(A R A+A S-P A-Q)+\operatorname{rk}(A R A)+\operatorname{rk}(Q) \leq 3 m .
$$

Since we had no conditions on the element

$$
\left(\begin{array}{ll}
P & Q \\
R & S
\end{array}\right) \in Y
$$

we also get $\operatorname{rk}\left(P^{\prime} A+\left(P^{\prime} A\right)^{T}\right) \leq 3 m$ for all

$$
\left(\begin{array}{cc}
P^{\prime} & \bullet \\
\bullet & \bullet
\end{array}\right) \in \mathrm{GL}_{n} \cdot\left(\begin{array}{ll}
P & Q \\
R & S
\end{array}\right) \subseteq Y
$$

and hence $\operatorname{rk}\left(P^{\prime} A+\left(P^{\prime} A\right)^{T}\right) \leq 3 m$ for all $P^{\prime} \sim P$. Now assume that $n>6 m$. Choose $A=\operatorname{Diag}\left(I_{2 m+1}, 0\right)$ and write

$$
P^{\prime}=\left(\begin{array}{ll}
P_{11}^{\prime} & P_{12}^{\prime} \\
P_{21}^{\prime} & P_{22}^{\prime}
\end{array}\right) \sim P
$$

with $P_{21}^{\prime} \in \mathfrak{g l}_{2 m+1}$. Then

$$
P^{\prime} A+\left(P^{\prime} A\right)^{T}=\left(\begin{array}{ccc}
\bullet & \bullet & P_{21}^{\prime T} \\
\bullet & & \\
P_{21}^{\prime} & &
\end{array}\right)
$$

and hence $\operatorname{rk}\left(P_{21}^{\prime}\right) \leq 3 m / 2$. By Proposition 16, we see that $\operatorname{rk}\left(P, I_{n}\right) \leq 3 m / 2$ and hence $\operatorname{rk}\left(P+\lambda I_{n}\right) \leq 3 m / 2$ for some $\lambda \in K$. Next, choose $A=I_{n}$. Then we see that $\operatorname{rk}\left(P+P^{T}\right) \leq 3 m$. So

$$
\operatorname{rk}\left(2 \lambda I_{n}\right) \leq \operatorname{rk}\left(P+P^{T}\right)+\operatorname{rk}\left(P+\lambda I_{n}\right)+\operatorname{rk}\left(P^{T}+\lambda I_{n}\right) \leq 6 m<n
$$

and hence $\lambda=0$. So we in fact have $\operatorname{rk}(P) \leq 3 \mathrm{~m} / 2$. In particular, we see that $\operatorname{rk}\left(M_{11}\right)=\operatorname{rk}\left(M_{22}\right) \leq 3 m / 2$. Combining this with $\operatorname{rk}\left(M_{12}\right), \operatorname{rk}\left(M_{21}\right) \leq m$, we get $\operatorname{rk}(M) \leq 5 m$.

Using Lemma 41, we see that there is an $m \in \mathbb{Z}_{\geq 0}$ such that

$$
X_{i} \subseteq\left\{\left(\begin{array}{ll}
P & Q \\
R & S
\end{array}\right) \in \mathfrak{s p}_{2 n} \mid \operatorname{rk}(P) \leq m\right\}
$$

for all $i \gg 0$. As in the proof of Lemma 30 , we see using Lemma 29 that this in fact holds for $m=0$. 
Lemma 42. Let $n \in \mathbb{N}$ and let $Y \subsetneq \mathfrak{s p}_{2 n}$ be an $\mathrm{Sp}_{2 n}$-stable closed subset of

$$
\left\{\left(\begin{array}{cc}
0 & Q \\
R & 0
\end{array}\right) \mid \begin{array}{c}
Q \in \mathfrak{g l}_{n}, Q=Q^{T} \\
R \in \mathfrak{g l}_{n}, R=R^{T}
\end{array}\right\}
$$

Then $Y \subseteq\{0\}$.

Proof. Let

$$
\left(\begin{array}{ll}
0 & Q \\
R & 0
\end{array}\right)
$$

be an element of $Y$. Then

$$
\left(\begin{array}{cc}
0 & I_{n} \\
-I_{n} & I_{n}
\end{array}\right)\left(\begin{array}{cc}
0 & Q \\
R & 0
\end{array}\right)\left(\begin{array}{cc}
0 & I_{n} \\
-I_{n} & I_{n}
\end{array}\right)^{-1}=\left(\begin{array}{ll}
R & \bullet \\
\bullet & \bullet
\end{array}\right) \in Y
$$

since $Y$ is $\mathrm{Sp}_{2 n}$-stable and therefore $R=0$. By Lemma 41, we see that $Q=0$.

The lemma shows that $X \subseteq\{0\}$. So when $\#\left\{i \mid l_{i}>1\right\}=\infty$, the only $G$-stable closed subsets of $V$ are $V,\{0\}$ and $\emptyset$. This proves in particular that $V$ is $G$-Noetherian.

\section{Limits OF ClASSiCAL GROUPS OF TYPE D}

Recall that we assume that $\operatorname{char}(K) \neq 2$. In this section, we let $G$ be the direct limit of a sequence

$$
\mathrm{O}_{2 n_{1}} \stackrel{\iota_{1}}{\longleftrightarrow} \mathrm{O}_{2 n_{2}} \stackrel{\iota_{2}}{\longleftrightarrow} \mathrm{O}_{2 n_{3}} \stackrel{\iota_{3}}{\longleftrightarrow \ldots}
$$

of diagonal embeddings given by

$$
\begin{aligned}
\iota_{i}: \mathrm{O}_{2 n_{i}} & \hookrightarrow \mathrm{O}_{2 n_{i+1}} \\
\left(\begin{array}{cc}
A & B \\
C & D
\end{array}\right) & \mapsto\left(\begin{array}{cc}
\operatorname{Diag}\left(A, \ldots, A, I_{z_{i}}\right) & \operatorname{Diag}(B, \ldots, B, 0) \\
\operatorname{Diag}(C, \ldots, C, 0) & \operatorname{Diag}\left(D, \ldots, D, I_{z_{i}}\right)
\end{array}\right)
\end{aligned}
$$

with $l_{i}$ blocks $A, B, C, D \in \mathfrak{g l}_{n_{i}}$ for some $l_{i} \in \mathbb{N}$ and $z_{i} \in \mathbb{Z}_{\geq 0}$. We let $V$ be the inverse limit of the sequence

$$
\mathfrak{D}_{2 n_{1}} \ll-\mathfrak{D}_{2 n_{2}} \nVdash-\mathfrak{D}_{2 n_{3}} \longleftarrow
$$

where the maps are given by

$$
\left(\begin{array}{cccccccc}
P_{11} & \ldots & P_{1 l_{i}} & \bullet & Q_{11} & \ldots & Q_{1 l_{i}} & \bullet \\
\vdots & & \vdots & \vdots & \vdots & & \vdots & \vdots \\
P_{l_{i} 1} & \ldots & P_{l_{i} l_{i}} & \vdots & Q_{l_{i} 1} & \ldots & Q_{l_{i} l_{i}} & \vdots \\
\bullet & \ldots & \ldots & \bullet & \bullet & \ldots & \ldots & \bullet \\
R_{11} & \ldots & R_{1 l_{i}} & \bullet & S_{11} & \ldots & S_{1 l_{i}} & \bullet \\
\vdots & & \vdots & \vdots & \vdots & & \vdots & \vdots \\
R_{l_{i} 1} & \ldots & R_{l_{i} l_{i}} & \vdots & S_{l_{i} 1} & \ldots & S_{l_{i} l_{i}} & \vdots \\
\bullet & \ldots & \ldots & \bullet & \bullet & \ldots & \ldots & \bullet
\end{array}\right) \mapsto\left(\begin{array}{ccc}
\sum_{k=1}^{l_{i}} P_{k k} & \sum_{k=1}^{l_{i}} Q_{k k} \\
\sum_{k=1}^{l_{i}} R_{k k} & \sum_{k=1}^{l_{i}} S_{k k}
\end{array}\right)
$$

with $P_{k \ell}=-S_{\ell k}^{T}, Q_{k \ell}, R_{k \ell} \in \mathrm{gI}_{n_{i}}$ such that $Q_{k \ell}+Q_{\ell k}^{T}=R_{k \ell}+R_{\ell k}^{T}=0$.

Theorem 43. The space $V$ is G-Noetherian. 
This proof of this theorem will have the same structure as the proof of Theorem 37. Let $X \subsetneq V$ be a $G$-stable closed subset. Let $X_{i}$ be the closure of the projection of $X$ to $\mathfrak{D}_{2 n_{i}}$ and let $I\left(X_{i}\right) \subseteq K\left[\mathfrak{v}_{2 n_{i}}\right]$ be the ideal of $X_{i}$. If $\#\left\{i \mid l_{i}>1\right\}<\infty$, then Theorem 43 follows from [ES, Theorem 1.2]. So we assume that $\#\left\{i \mid l_{i}>1\right\}=\infty$. By restricting to an infinite subsequence, we may assume that $l_{i} \geq 3$ for all $i \in \mathbb{N}$.

Lemma 44. Let $n \in \mathbb{N}$, let $Y \subsetneq \mathbb{D}_{2 n}$ be an $\mathrm{O}_{2 n}$-stable closed subset and let $\mathrm{Z}$ be the closed subset

$$
\left\{\left(\begin{array}{cc}
P & Q \\
R & -P^{T}
\end{array}\right) \in \mathfrak{s p}_{2 n} \mid P=P^{T}\right\}
$$

of $\mathfrak{D}_{2 n}$. Then there is a non-zero polynomial $f \in I(Y)$ whose top-graded part is not contained in the ideal of $\mathrm{Z}$.

Proof. The proof is analogous to the proof of Lemma 39

Lemma 45. Let $i \in \mathbb{N}$ and let $f=f(P, Q, R) \in I\left(X_{i}\right)$ be a non-zero polynomial whose top-graded part $g$ is not contained in the ideal of

$$
\left\{\left(\begin{array}{cc}
P & Q \\
R & -P^{T}
\end{array}\right) \in \mathfrak{v}_{2 n_{i}} \mid P=P^{T}\right\}
$$

Then $I\left(X_{i+1}\right) \cap K\left[r_{k \ell} \mid 1 \leq k, \ell \leq n_{i+1}\right] /\left(r_{k \ell}+r_{\ell k}\right)$ contains a non-zero polynomial with degree at most $\operatorname{deg}(f)$.

Proof. The proof is analogous to the proof of Lemma 40, replacing $A(\lambda)$ by the matrix

$$
\left(\begin{array}{cccccc}
I_{m} & & & & \lambda I_{m} & \\
& I_{m} & & -\lambda I_{m} & & \\
& & I_{n-2 m} & & & \\
& & & I_{m} & & \\
& & & & I_{m} & \\
& & & & & I_{n-2 m}
\end{array}\right) \in \mathrm{O}_{2 n} .
$$

Since $X \subsetneq V$, we know that $X_{j} \subsetneq \mathfrak{D}_{2 n_{j}}$ for some $j \in \mathbb{N}$. Using the previous lemma, we see that there is a $d \in \mathbb{Z}_{\geq 0}$ such that $I\left(X_{i}\right) \cap K\left[r_{k \ell} \mid 1 \leq k, \ell \leq n_{i}\right] /\left(r_{k \ell}+r_{\ell k}\right)$ contains a non-zero polynomial of degree at most $d$ for all $i>j$.

Lemma 46. Let $n \in \mathbb{N}$, let $Y \subsetneq \mathfrak{D}_{2 n}$ be an $\mathrm{O}_{2 n}$-stable closed subset and suppose that

$$
I(Y) \cap K\left[r_{k \ell} \mid 1 \leq k, \ell \leq n\right] /\left(r_{k \ell}+r_{\ell k}\right)
$$

contains a non-zero polynomial of degree $m+1$. Then

$$
Y \subseteq\left\{\left(\begin{array}{ll}
P & Q \\
R & S
\end{array}\right) \in \mathfrak{v}_{2 n} \mid \operatorname{rk}(Q), \operatorname{rk}(R) \leq 2 m\right\} .
$$

Furthermore, if $n \geq 20 m+2$, then $\operatorname{rk}(M) \leq 10 m$ for all $M \in Y$.

Proof. Let $Z$ be the closure of the subset

$$
\left\{R \mid\left(\begin{array}{ll}
P & Q \\
R & S
\end{array}\right) \in Y\right\}
$$


of $\left\{R \in \mathfrak{g l}_{n} \mid R+R^{T}=0\right\}$. Let $\mathrm{GL}_{n}$ act on $\mathfrak{D}_{2 n}$ via the diagonal embedding

$$
\begin{aligned}
\mathrm{GL}_{n} & \hookrightarrow \mathrm{O}_{2 n} \\
g & \mapsto \operatorname{Diag}\left(g, g^{-T}\right)
\end{aligned}
$$

and on $\left\{R \in \mathrm{gI}_{n} \mid R+R^{T}=0\right\}$ by $g \cdot R=g R g^{T}$. Then we see that $Y$ is $\mathrm{GL}_{n}$-stable and therefore $Z$ is also $\mathrm{GL}_{n}$-stable. So $Z$ must consist of all skew-symmetric matrices of rank at most $h$ for some even $h \leq n$. Since $I(Z)$ contains a non-zero polynomial of degree $m+1$, we see that $h \leq 2 m$. See [ADF, §3]. So

$$
Y \subseteq\left\{\left(\begin{array}{ll}
P & Q \\
R & S
\end{array}\right) \in \mathfrak{v}_{2 n} \mid \operatorname{rk}(R) \leq 2 m\right\} .
$$

Let $A \in \mathfrak{g l}_{n}$ be a skew-symmetric matrix and let

$$
\left(\begin{array}{ll}
P & Q \\
R & S
\end{array}\right)
$$

be an element of $Y$. Then we have

$$
\left(\begin{array}{cc}
0 & I_{n} \\
I_{n} & A
\end{array}\right) \in \mathrm{O}_{2 n}
$$

and hence

$$
\left(\begin{array}{cc}
0 & I_{n} \\
I_{n} & A
\end{array}\right)\left(\begin{array}{ll}
P & Q \\
R & S
\end{array}\right)\left(\begin{array}{cc}
0 & I_{n} \\
I_{n} & A
\end{array}\right)^{-1}=\left(\begin{array}{cc}
\bullet & \bullet \\
Q+A S-P A-A R A & \bullet
\end{array}\right) \in Y .
$$

So we get $\operatorname{rk}(Q+A S-P A-A R A) \leq 2 m$. Choosing $A=0$, we see that

$$
Y \subseteq\left\{\left(\begin{array}{ll}
P & Q \\
R & S
\end{array}\right) \in \mathfrak{v}_{2 n} \mid \operatorname{rk}(Q) \leq 2 m\right\} .
$$

Assume that $n \geq 2(3 m+1)$. Since $S=-P^{T}$ and $A=-A^{T}$, we get

$$
\operatorname{rk}\left(P A-(P A)^{T}\right) \leq \operatorname{rk}(Q+A S-P A-A R A)+\operatorname{rk}(A R A)+\operatorname{rk}(Q) \leq 6 m .
$$

Since $Y$ is $\mathrm{GL}_{n}$-stable, we have $\operatorname{rk}\left(P^{\prime} A-\left(P^{\prime} A\right)^{T}\right) \leq 6 m$ for all $P^{\prime} \sim P$. Choose

$$
A=\left(\begin{array}{ccc} 
& I_{3 m+1} \\
-I_{3 m+1} & &
\end{array}\right)
$$

and write

$$
P^{\prime}=\left(\begin{array}{lll}
P_{11}^{\prime} & P_{12}^{\prime} & P_{13}^{\prime} \\
P_{21}^{\prime} & P_{22}^{\prime} & P_{23}^{\prime} \\
P_{31}^{\prime} & P_{32}^{\prime} & P_{33}^{\prime}
\end{array}\right)
$$

with $P_{11}^{\prime}, P_{13}^{\prime}, P_{31}^{\prime}, P_{33}^{\prime} \in \mathfrak{g l}_{3 m+1}$. Then

$$
P^{\prime} A-\left(P^{\prime} A\right)^{T}=\left(\begin{array}{ccc}
\bullet & P_{23}^{\prime T} & \bullet \\
-P_{23}^{\prime} & 0 & P_{21}^{\prime} \\
\bullet & -P_{21}^{\prime T} & \bullet
\end{array}\right)
$$

has rank at most $6 \mathrm{~m}$. Therefore the submatrix

$$
\left(\begin{array}{cc}
0 & P_{21}^{\prime} \\
-P_{21}^{\prime T} & \bullet
\end{array}\right)
$$


also has rank at most $6 m$ and hence and hence $\operatorname{rk}\left(P_{21}^{\prime}\right) \leq 3 m$. By Proposition 16, we see that $\operatorname{rk}\left(P, I_{n}\right) \leq 3 m$. Hence

$$
Y \subseteq\left\{M \in \mathfrak{b}_{2 n} \mid \operatorname{rk}\left(M, \operatorname{Diag}\left(I_{n},-I_{n}\right)\right) \leq 2 \cdot 2 m+2 \cdot 3 m=10 m\right\} .
$$

Assume that $n \geq 20 m+2$, let $M+\lambda \operatorname{Diag}\left(I_{n},-I_{n}\right)$ be an element of $Y$ with $\operatorname{rk}(M) \leq 10 m$ and $\lambda \in K$ and let $B \in \mathfrak{g l}_{n}$ be a skew-symmetric matrix of rank at least $n-1$. Then

$$
\left(\begin{array}{cc}
I_{n} & B \\
& I_{n}
\end{array}\right) \in \mathrm{O}_{2 n}
$$

and therefore

$$
\left(\begin{array}{cc}
I_{n} & B \\
& I_{n}
\end{array}\right)\left(M+\lambda \operatorname{Diag}\left(I_{n},-I_{n}\right)\right)\left(\begin{array}{cc}
I_{n} & B \\
& I_{n}
\end{array}\right)^{-1} \in Y
$$

So this element must be of the form $M^{\prime}-\mu \operatorname{Diag}\left(I_{n},-I_{n}\right)$ with $\operatorname{rk}(M) \leq 10 m$ and $\mu \in K$. Now note that

$$
\operatorname{rk}\left(\lambda\left(\begin{array}{cc}
I_{n} & B \\
& I_{n}
\end{array}\right) \operatorname{Diag}\left(I_{n},-I_{n}\right)\left(\begin{array}{cc}
I_{n} & B \\
& I_{n}
\end{array}\right)^{-1}+\mu \operatorname{Diag}\left(I_{n},-I_{n}\right)\right) \leq \operatorname{rk}(M)+\operatorname{rk}\left(M^{\prime}\right) \leq 20 m
$$

So since

$$
\lambda\left(\begin{array}{cc}
I_{n} & B \\
& I_{n}
\end{array}\right) \operatorname{Diag}\left(I_{n},-I_{n}\right)\left(\begin{array}{cc}
I_{n} & B \\
& I_{n}
\end{array}\right)^{-1}+\mu \operatorname{Diag}\left(I_{n},-I_{n}\right)=\left(\begin{array}{cc}
\bullet & -2 \lambda B \\
\bullet & \bullet
\end{array}\right)
$$

and $\operatorname{rk}(2 B) \geq n-1>20 m$, we see that $\lambda=0$. Hence $Y$ consists of matrices of rank at most $10 \mathrm{~m}$.

Using Lemma 46, we see that there is an $m \in \mathbb{Z}_{\geq 0}$ such that

$$
X_{i} \subseteq\left\{\left(\begin{array}{ll}
P & Q \\
R & S
\end{array}\right) \in \mathfrak{o}_{2 n} \mid \operatorname{rk}(P) \leq m\right\}
$$

for all $i \gg 0$. As in the proof of Lemma 30 , we see using Lemma 29 that this in fact holds for $m=0$.

Lemma 47. Let $n \in \mathbb{N}$ and let $Y \subsetneq \mathbb{D}_{2 n}$ be an $\mathrm{O}_{2 n}$-stable closed subset of

$$
\left\{\left(\begin{array}{cc}
0 & Q \\
R & 0
\end{array}\right) \mid \begin{array}{c}
Q \in \mathfrak{g l}_{n}, Q+Q^{T}=0 \\
R \in \mathfrak{g l}_{n}, R+R^{T}=0
\end{array}\right\}
$$

Then $Y \subseteq\{0\}$.

Proof. Let

$$
\left(\begin{array}{ll}
0 & Q \\
R & 0
\end{array}\right)
$$

be an element of $Y$. Then

$$
\left(\begin{array}{ll}
I_{n} & A \\
& I_{n}
\end{array}\right)\left(\begin{array}{ll}
0 & Q \\
R & 0
\end{array}\right)\left(\begin{array}{ll}
I_{n} & A \\
& I_{n}
\end{array}\right)^{-1}=\left(\begin{array}{cc}
A R & \bullet \\
\bullet & \bullet
\end{array}\right) \in Y
$$

for all $A \in \mathrm{gI}_{n}$ with $A+A^{T}=0$ since $Y$ is $\mathrm{O}_{2 n}$-stable and therefore $R=0$. By Lemma46, we see that $Q=0$.

As in the previous section, the lemma shows that $X \subseteq\{0\}$. So again, when $\#\left\{i \mid l_{i}>1\right\}=\infty$, the only $G$-stable closed subsets of $V$ are $V,\{0\}$ and $\emptyset$ and the space $V$ is G-Noetherian. 


\section{Limits OF CLASSiCAL GROUPS OF TYPE B}

In this last section of the proof of the Main Theorem, we still assume that $\operatorname{char}(K) \neq 2$. Now, we let $G$ be the direct limit of a sequence

$$
\mathrm{O}_{2 n_{1}+1} \stackrel{l_{1}}{\longleftrightarrow} \mathrm{O}_{2 n_{2}+1} \stackrel{l_{2}}{\longleftrightarrow} \mathrm{O}_{2 n_{3}+1} \stackrel{l_{3}}{\longleftrightarrow \ldots}
$$

of diagonal embeddings. To prove that the corresponding inverse limit $V$ is $G$ Noetherian, it suffices to consider the case where $K$ is algebraically closed. The following proposition shows that, if $K=\bar{K}$ and $\iota_{i}$ has signature $\left(l_{i}, z_{i}\right)$ with $l_{i}$ even, then we can insert a group of type $D$ into the sequence defining $G$.

Proposition 48. Suppose that $K$ is algebraically closed. Let $m, n \in \mathbb{Z}_{\geq 0}$ be integers and let $\iota: \mathrm{O}_{2 m+1} \hookrightarrow \mathrm{O}_{2 n+1}$ be a diagonal embedding with signature $(l, z)$. If l is even, then $\iota$ is the composition of diagonal embeddings $\mathrm{O}_{2 m+1} \hookrightarrow \mathrm{O}_{l(2 m+1)}$ and $\mathrm{O}_{l(2 m+1)} \hookrightarrow \mathrm{O}_{2 n+1}$.

Proof. By Lemma11, it suffices to find one diagonal embedding $\iota: \mathrm{O}_{2 m+1} \hookrightarrow \mathrm{O}_{2 n+1}$ with signature $(l, z)$ for which the proposition holds. For $k \in \mathbb{N}$, note that the group

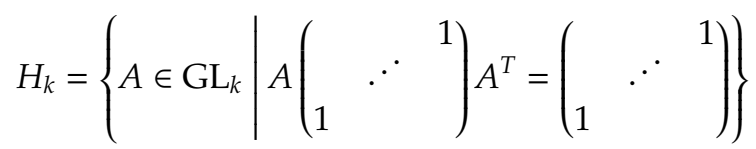

is conjugate to $\mathrm{O}_{k}$ in $\mathrm{GL}_{k}$. The map

$$
\begin{aligned}
H_{2 m+1} & \hookrightarrow H_{l(2 m+1)} \\
A & \mapsto \operatorname{Diag}(A, \ldots, A)
\end{aligned}
$$

induces a diagonal embedding $\mathrm{O}_{2 m+1} \hookrightarrow \mathrm{O}_{l(2 m+1)}$ with signature $(l, 0)$. Note that $2 n+1=l(2 m+1)+z$ and so $z$ is odd. Write $z=2 k+1$. Then the map

$$
\begin{aligned}
\mathrm{O}_{l(2 m+1)} & \mapsto \mathrm{O}_{2 n+1} \\
\left(\begin{array}{ll}
A & B \\
C & D
\end{array}\right) & \mapsto\left(\begin{array}{lllll}
A & & & B & \\
& I_{k} & & & \\
& & 1 & & \\
C & & & D & \\
& & & & I_{k}
\end{array}\right)
\end{aligned}
$$

is a diagonal embedding with signature $(1, z)$. Now, let $\iota$ be the composition of these two diagonal embeddings. Then $\iota$ is itself a diagonal embedding and has signature $(l, z)$.

Suppose that $K$ is algebraically closed and that the diagonal embeddings $\iota_{i}$ have signatures $\left(l_{i}, z_{i}\right)$ with $l_{i}$ even for infinitely many $i \in \mathbb{N}$. Then the proposition shows that we can replace our sequence by a supersequence in which groups of type $D$ appear infinitely many times. In this case $V$ is $G$-Noetherian by the previous section. So, even if $K$ is not algebraically closed, we only have to consider the case where this does not happen. And, by replacing our sequence by an infinite subsequence, we may assume that $l_{i} \in \mathbb{N}$ odd for every $i \in \mathbb{N}$. As both $n_{i}$ and $n_{i+1}=l_{i} n_{i}+z_{i}$ are odd, this forces $z_{i} \in \mathbb{Z}_{\geq 0}$ to be even for all $i \in \mathbb{N}$. Our next task is to find diagonal embeddings with such signatures. 
First, note that for $n \in \mathbb{N}$ and $z \in \mathbb{Z}_{\geq 0}$ the map

$$
\begin{aligned}
\iota_{1,2 z}: \mathrm{O}_{2 n+1} & \hookrightarrow \mathrm{O}_{2(n+z)+1} \\
\left(\begin{array}{lll}
A & \alpha & B \\
\beta & \mu & \gamma \\
C & \delta & D
\end{array}\right) & \mapsto\left(\begin{array}{lllll}
A & & \alpha & B & \\
& I_{z} & & & \\
\beta & & \mu & \gamma & \\
C & & \delta & D & \\
& & & & I_{z}
\end{array}\right)
\end{aligned}
$$

is a diagonal embedding with signature $(1,2 z)$. Here $A, B, C, D \in \mathfrak{g l}_{n}, \alpha, \beta^{T}, \gamma^{T}, \delta \in K^{n}$ and $\mu \in K$. The associated map of Lie algebras is

$$
\begin{aligned}
\mathrm{pr}_{1,2 z}: & \mathfrak{o}_{2(n+z)+1} \\
\left(\begin{array}{ccccc}
P & \bullet & v & Q & \bullet \\
\bullet & \bullet & \bullet & \bullet & \bullet \\
\phi & \bullet & 0 & \psi & \bullet \\
R & \bullet & w & S & \bullet \\
\bullet & \bullet & \bullet & \bullet & \bullet
\end{array}\right) & \mapsto\left(\begin{array}{ccc}
P & v & Q \\
\phi & 0 & \psi \\
R & w & S
\end{array}\right)
\end{aligned}
$$

with $P=-S^{T}, Q, R \in \mathfrak{g l}_{n}$ and $v=-\psi^{T}, w=-\phi^{T} \in K^{n}$ such that $Q+Q^{T}=R+R^{T}=0$.

Next, we construct a diagonal embedding $\mathrm{O}_{2 n+1} \hookrightarrow \mathrm{O}_{l(2 n+1)}$ with signature $(l, 0)$ for all $n \in \mathbb{N}$ and $l \in \mathbb{N}$ odd. Write

$$
J_{k}=\left(\begin{array}{ll} 
& \cdot \\
1 &
\end{array}\right) \in \mathrm{GL}_{k}
$$

for $k \in \mathbb{N}$ and take

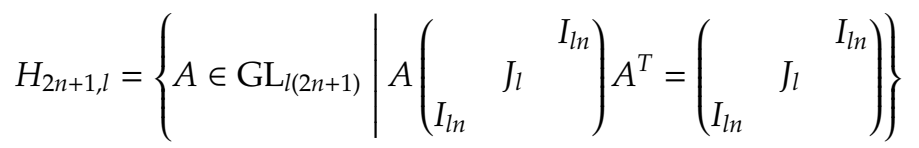

for all $n \in \mathbb{N}$ and $l \in \mathbb{N}$ odd. Then we have

$$
P\left(\begin{array}{lll} 
& J_{l} & I_{l n} \\
I_{l n} & &
\end{array}\right) P^{T}=\left(\begin{array}{lll} 
& & I_{l n+k} \\
I_{l n+k} & &
\end{array}\right)
$$

where

$$
P=\left(\begin{array}{lllll}
I_{l n} & & & & \\
& I_{k} & & & \\
& & 1 & & \\
& & & & I_{l n} \\
& & & J_{k} &
\end{array}\right)
$$

is a permutation matrix. So the map

$$
\begin{aligned}
H_{2 n+1, l} & \rightarrow \mathrm{O}_{l(2 n+1)} \\
A & \mapsto P A P^{T}
\end{aligned}
$$


is an isomorphism. Consider the map

$$
\begin{aligned}
& \mathrm{O}_{2 n+1} \hookrightarrow H_{2 n+1, l} \\
& \left(\begin{array}{ccc}
A & \alpha & B \\
\beta & \mu & \gamma \\
C & \delta & D
\end{array}\right) \mapsto\left(\begin{array}{cccccccccc}
A & & & \alpha & & & & & B \\
& \ddots & & & \ddots & & & . & \\
& & A & & & \alpha & B & & \\
\beta & & & \mu & & & & & \gamma \\
& \ddots & & & \ddots & & & . & \\
& & \beta & & & \mu & \gamma & & \\
& & C & & & \delta & D & & \\
& . & & & . & & & \ddots & \\
C & & & \delta & & & & & D
\end{array}\right)
\end{aligned}
$$

where $A, B, C, D \in \mathfrak{g I}_{n}, \alpha, \beta^{T}, \gamma^{T}, \delta \in K^{n}$ and $\mu \in K$ all occur $l$ times on the right hand side. Write $l=2 k+1$. By taking the composition of these two maps, we get a diagonal embedding $\mathrm{O}_{2 n+1} \hookrightarrow \mathrm{O}_{l(2 n+1)}$ with signature $(l, 0)$.

Write $J=J_{l}$ and consider the Lie algebra

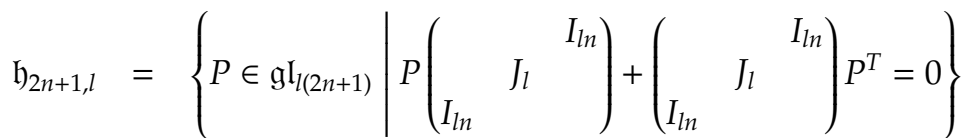

$$
\begin{aligned}
& =\left\{\left(\begin{array}{ccc}
P & V & Q \\
\Phi & U & \Psi \\
R & W & S
\end{array}\right) \in \operatorname{gl}_{l(2 n+1)} \quad \begin{array}{c}
P+S^{T}=Q+Q^{T}=R+R^{T}=0 \\
V J+\Psi^{T}=W J+\Phi^{T}=0 \\
U J+J U^{T}=0
\end{array}\right\}
\end{aligned}
$$

of $H_{2 n+1, l}$. The map $\mathrm{O}_{2 n+1} \hookrightarrow H_{2 n+1, l}$ corresponds to the map $\mathfrak{h}_{2 n+1, l} \rightarrow \mathfrak{v}_{2 n+1}$ sending

$$
\left(\begin{array}{ccccccccc}
P_{11} & \ldots & P_{1 l} & V_{11} & \ldots & V_{1 l} & Q_{11} & \ldots & Q_{1 l} \\
\vdots & & \vdots & \vdots & & \vdots & \vdots & & \vdots \\
P_{l 1} & \ldots & P_{l l} & V_{l 1} & \ldots & V_{l l} & Q_{l 1} & \ldots & Q_{l l} \\
\Phi_{11} & \ldots & \Phi_{1 l} & U_{11} & \ldots & U_{1 l} & \Psi_{11} & \ldots & \Psi_{1 l} \\
\vdots & & \vdots & \vdots & & \vdots & \vdots & & \vdots \\
\Phi_{l 1} & \ldots & \Phi_{l l} & U_{l 1} & \ldots & U_{l l} & \Psi_{l 1} & \ldots & \Psi_{l l} \\
R_{11} & \ldots & R_{1 l} & W_{11} & \ldots & W_{1 l} & S_{11} & \ldots & S_{1 l} \\
\vdots & & \vdots & \vdots & & \vdots & \vdots & & \vdots \\
R_{l 1} & \ldots & R_{l l} & W_{l 1} & \ldots & W_{l l} & S_{l 1} & \ldots & S_{l l}
\end{array}\right)
$$

to

$$
\left(\begin{array}{ccc}
P_{11}+\cdots+P_{l l} & V_{11}+\cdots+V_{l l} & Q_{1 l}+\cdots+Q_{l 1} \\
\Phi_{11}+\cdots+\Phi_{l l} & U_{11}+\cdots+U_{l l} & \Psi_{1 l}+\cdots+\Psi_{l 1} \\
R_{1 l}+\cdots+R_{l 1} & W_{1 l}+\cdots+W_{l 1} & S_{11}+\cdots+S_{l l}
\end{array}\right) .
$$

Here, for each entry, we either sum along the diagonal or along the anti-diagonal in a manner consistent with the definition of the map $\mathrm{O}_{2 n+1} \hookrightarrow H_{2 n+1, l}$. The map $H_{2 n+1, l} \rightarrow \mathrm{O}_{l(2 n+1)}$ corresponds to the map $\mathfrak{v}_{l(2 n+1)} \rightarrow \mathfrak{h}_{2 n+1, l}$ sending $Q$ to $P^{T} Q P^{-T}$.

We let the diagonal embeddings in the sequence

$$
\mathrm{O}_{2 n_{1}+1} \stackrel{\iota_{1}}{\longleftrightarrow} \mathrm{O}_{2 n_{2}+1} \stackrel{\iota_{2}}{\longleftrightarrow} \mathrm{O}_{2 n_{3}+1} \stackrel{\iota_{3}}{\longleftrightarrow \ldots}
$$


be (compositions of) the forms above. As in the previous sections, if only finitely many embeddings have signature $\left(l_{i}, 2 z_{i}\right)$ with $l_{i}>1$, then Theorem 37 follows from [ES. Theorem 1.2]. So we assume that $\#\left\{l_{i} \mid l_{i}>1\right\}=\infty$. Now, by replacing our sequence by an infinite subsequence, we may assume that $l_{i} \in \mathbb{N}$ is odd and at least 3 for every $i \in \mathbb{N}$.

Lemma 49. Let $Y \subsetneq \mathfrak{h}_{2 n+1, l}$ be an $H_{2 n+1, l}$-stable closed subset and let $Z$ be the closed subset

$$
\left\{\left(\begin{array}{ccc}
P & V & Q \\
\Phi & U & \Psi \\
R & W & S
\end{array}\right) \in \mathfrak{h}_{2 n+1, l} \mid P=P^{T}\right\}
$$

of $\mathfrak{h}_{2 n+1, l}$. Then there is a non-zero polynomial $f \in I(Y)$ whose top-graded part is not contained in the ideal of $Z$.

Proof. The proof is analogous to the proof of Lemma 39

Lemma 50. Let $X$ be an $H_{2 n+1, l}$-stable closed subset of $\mathfrak{h}_{2 n+1, l}$ and let $Y$ be the closure of its image in $\mathfrak{o}_{2 n+1}$. Let $f \in I(Y) \subseteq K\left[\mathrm{v}_{2 n+1}\right]$ be a non-zero polynomial whose top-graded part $g$ is not contained in the ideal of

$$
\left\{\left(\begin{array}{ccc}
P & V & Q \\
\Phi & U & \Psi \\
R & W & S
\end{array}\right) \in \mathfrak{h}_{2 n+1, l} \mid P=P^{T}\right\} .
$$

Then $I(X)$ contains a non-zero polynomial with degree at most $\operatorname{deg}(f)$ that only depends on $R$ and two columns of $W$.

Proof. Consider the matrix

$$
\left(\begin{array}{ccccccccc}
P_{11} & \ldots & P_{1 l} & V_{11} & \ldots & V_{1 l} & Q_{11} & \ldots & Q_{1 l} \\
\vdots & & \vdots & \vdots & & \vdots & \vdots & & \vdots \\
P_{l 1} & \ldots & P_{l l} & V_{l 1} & \ldots & V_{l l} & Q_{l 1} & \ldots & Q_{l l} \\
\Phi_{11} & \ldots & \Phi_{1 l} & U_{11} & \ldots & U_{1 l} & \Psi_{11} & \ldots & \Psi_{1 l} \\
\vdots & & \vdots & \vdots & & \vdots & \vdots & & \vdots \\
\Phi_{l 1} & \ldots & \Phi_{l l} & U_{l 1} & \ldots & U_{l l} & \Psi_{l 1} & \ldots & \Psi_{l l} \\
R_{11} & \ldots & R_{1 l} & W_{11} & \ldots & W_{1 l} & S_{11} & \ldots & S_{1 l} \\
\vdots & & \vdots & \vdots & & \vdots & \vdots & & \vdots \\
R_{l 1} & \ldots & R_{l l} & W_{l 1} & \ldots & W_{l l} & S_{l 1} & \ldots & S_{l l}
\end{array}\right) \in \mathfrak{h}_{2 n+1, l}
$$

and note that the polynomial $f=f(P, Q, R, v, w) \in I(Y)$ induces the element

$$
f\left(P_{11}+\cdots+P_{l l}, Q_{1 l}+\cdots+Q_{l 1}, R_{1 l}+\cdots+R_{l 1}, V_{11}+\cdots+V_{l l}, W_{1 l}+\cdots+W_{l 1}\right)
$$

of $I(X)$. Consider the matrix

$$
A(\lambda)=\left(\begin{array}{ccccccc}
I_{n} & & & & & & -\lambda I_{n} \\
& \ddots & & & & & \\
& & I_{n} & & \lambda I_{n} & & \\
& & & I_{l} & & & \\
& & & & I_{n} & & \\
& & & & & \ddots & \\
& & & & & & I_{n}
\end{array}\right) \in H_{2 n+1, l}
$$


for $\lambda \in K$. One can check that

$$
g\left(R_{1 l}-R_{l 1},-\left(R_{1 l}+R_{l 1}\right), R_{1 l}+\cdots+R_{l 1}, W_{1 l}-W_{l 1}, W_{1 l}+\cdots+W_{l 1}\right)
$$

is contained in the span of

$$
A(\lambda) \cdot f\left(P_{11}+\cdots+P_{l l}, Q_{1 l}+\cdots+Q_{l 1}, R_{1 l}+\cdots+R_{l 1}, V_{11}+\cdots+V_{l l}, W_{1 l}+\cdots+W_{l 1}\right)
$$

over all $\lambda \in K$. So it is an element of $I(X)$ and its degree is at most $\operatorname{deg}(f)$.

Next, consider the matrix

$$
B(\mu)=\left(\begin{array}{cccccc}
I_{l n} & & & & & \\
& 1 & & & & \\
& \mu & \ddots & & & \\
& & & \ddots & & \\
& & & -\mu & 1 & \\
& & & & & I_{l n}
\end{array}\right) \in H_{2 n+1, l}
$$

for $\mu \in K$. Let $h(P, Q, R, v, w)$ be the top-graded part of $g$ with respect to the grading where $P, Q, R$ get grading 0 and $v, w$ get grading 1 . Then one can check that

$$
h\left(R_{1 l}-R_{l 1},-\left(R_{1 l}+R_{l 1}\right), R_{1 l}+\cdots+R_{l 1},-W_{l-1,2}, W_{1 l}+W_{l-1,2}\right)
$$

is contained in the span of

$$
B(\mu) \cdot g\left(R_{1 l}-R_{l 1},-\left(R_{1 l}+R_{l 1}\right), R_{1 l}+\cdots+R_{l 1}, W_{1 l}-W_{l 1}, W_{1 l}+\cdots+W_{l 1}\right)
$$

over all $\mu \in K$. This polynomial is contained in $I(X)$ and has degree at most $\operatorname{deg}(f)$.

The following proposition tells us how to use the equation we gain from Lemma 49. Let $\mathrm{GL}_{n}$ act on $\left\{Q \in \mathrm{gl}_{n} \mid Q=-Q^{T}\right\}$ by $g \cdot Q=g Q g^{T}$. Let $k \leq n$ be an integer and let $\mathrm{GL}_{n}$ act on $K^{n \times k}$ by left-multiplication.

Proposition 51. Let $R \in \mathfrak{g l}_{n}$ be a skew-symmetric matrix and let $W \in K^{n \times k}$ be a matrix of rank $k$. Then the closure of the $\mathrm{GL}_{n}$-orbit of $(R, W)$ inside $\left\{Q \in \mathfrak{g l}_{n} \mid Q=-Q^{T}\right\} \oplus K^{n \times k}$ contains all tuples $(Q, V)$ with $\operatorname{rk}(Q) \leq \operatorname{rk}(R)-2 k$.

Proof. We will prove the proposition using induction on $k$. The case $k=0$ is wellknown. So assume that $0<2 k \leq \operatorname{rk}(R)$. Let $X$ be the closure of the $\mathrm{GL}_{n}$-orbit of $(R, W)$. Note that we may replace $(R, W)$ with any element in its $\mathrm{GL}_{n}$-orbit. Since $\operatorname{rk}(W)=k$, we may therefore assume that the last column of $W$ equals $e_{n}$. Now, if we act with a matrix of the form

$$
\left(\begin{array}{cccc}
1 & & & \\
& \ddots & & \\
& & \ddots & \\
a_{1} & \ldots & a_{n-1} & 1
\end{array}\right),
$$

then the last column of $W$ stays equal to $e_{n}$. And, the last column of $R$ becomes

$$
\left(\begin{array}{c}
a_{1} r_{1}+\cdots+a_{n-1} r_{n-1}+r_{n} \\
0
\end{array}\right)
$$


if we write

$$
R=\left(\begin{array}{cccc}
r_{1} & \ldots & r_{n-1} & r_{n} \\
\bullet & \ldots & \bullet & 0
\end{array}\right)
$$

with $r_{1}, \ldots, r_{n} \in K^{n-1}$. As $\operatorname{rk}(R)>k=\operatorname{rk}(W)$ and $e_{n}$ is contained in the image of $W$, we see that

$$
\left(\begin{array}{c}
a_{1} r_{1}+\cdots+a_{n-1} r_{n-1}+r_{n} \\
0
\end{array}\right)
$$

is not contained in the image of $W$ for some $a_{1}, \ldots, a_{n-1}$. So we may also assume that the last column of $R$ is not contained in the image of $W$. Next, note that the last column of $W$ stays $e_{n}$ and the last column of $R$ stays outside the image of $W$ if we act with a matrix of the form $\operatorname{Diag}(g, 1)$ with $g \in \mathrm{GL}_{n-1}$. Since the last column of $R$ is non-zero, we may therefore assume in addition that

$$
R=\left(\begin{array}{ccc}
R^{\prime} & w & 0 \\
-w^{T} & 0 & 1 \\
0 & -1 & 0
\end{array}\right)
$$

for some $R^{\prime} \in \mathfrak{g l}_{n-2}$ and $w \in K^{n-2}$. So the vector $e_{n-1}$ is not contained in the image of $W$. Note that $\operatorname{rk}\left(R^{\prime}\right) \geq \operatorname{rk}(R)-2$. Write

$$
W=\left(\begin{array}{ll}
W^{\prime} & 0 \\
v^{T} & 0 \\
u^{T} & 1
\end{array}\right)
$$

with $W^{\prime} \in K^{(n-2) \times(k-1)}$ and $u, v \in K^{k-1}$. Since $e_{n-1}$ is not contained in the image of $W$, the matrix $\left(W e_{n-1}\right)$ has rank $k+1$ and hence $\operatorname{rk}\left(W^{\prime}\right)=k-1$. The limit

$$
\lim _{\lambda \rightarrow 0} \operatorname{Diag}\left(I_{n-2}, \lambda, 1\right) \cdot(R, W)=\left(\left(\begin{array}{ccc}
R^{\prime} & 0 & 0 \\
0 & 0 & 0 \\
0 & 0 & 0
\end{array}\right),\left(\begin{array}{cc}
W^{\prime} & 0 \\
0 & 0 \\
u^{T} & 1
\end{array}\right)\right)
$$

is an element of $X$. Using the induction hypothesis, we see that $X$ contains

$$
\left(\left(\begin{array}{ccc}
Q & 0 & 0 \\
0 & 0 & 0 \\
0 & 0 & 0
\end{array}\right),\left(\begin{array}{cc}
V & 0 \\
0 & 0 \\
u^{T} & 1
\end{array}\right)\right)
$$

for all skew-symmetric matrices $Q \in \mathrm{gl}_{n-2}$ of rank at most $\operatorname{rk}(R)-2 k$ and all $V \in K^{(n-2) \times(k-1)}$. By acting with a permutation matrix, we see in particular that

$$
\left(\left(\begin{array}{ccc}
Q & 0 & 0 \\
0 & 0 & 0 \\
0 & 0 & 0
\end{array}\right),\left(\begin{array}{cc}
0 & 0 \\
I_{k-1} & 0 \\
u^{T} & 1
\end{array}\right)\right) \in X
$$

for all skew-symmetrix matrices $Q \in \mathfrak{g l}_{n-k}$ of rank at most $\operatorname{rk}(R)-2 k$. Therefore

$$
(\operatorname{Diag}(Q, 0), V)=\lim _{\lambda \rightarrow 0}\left(\operatorname{Diag}\left(I_{n-k}, \lambda I_{k}\right)+\left(0 V\left(\begin{array}{cc}
I_{k-1} & 0 \\
-u^{T} & 1
\end{array}\right)\right)\right) \cdot\left(\left(\begin{array}{ccc}
Q & 0 & 0 \\
0 & 0 & 0 \\
0 & 0 & 0
\end{array}\right),\left(\begin{array}{cc}
0 & 0 \\
I_{k-1} & 0 \\
u^{T} & 1
\end{array}\right)\right) \in X
$$

for all skew-symmetrix matrices $Q \in \mathfrak{g l}_{n-k}$ of rank at most $\mathrm{rk}(R)-2 k$ and all matrices $V \in K^{n \times k}$. So since $X$ is $\mathrm{GL}_{n}$-stable, we see that $(Q, V) \in X$ for all skew-symmetric matrices $Q \in \mathfrak{g l}_{n}$ of rank at most $\operatorname{rk}(R)-2 k$ and all matrices $V \in K^{n \times k}$. 
Lemma 52. There are integers $c_{0}, c_{1}, c_{2} \in \mathbb{N}$ such that the following holds: let $m \in \mathbb{Z}_{\geq 0}$ be an integer with $c_{2} m \leq n$ and let $M \in \mathfrak{h}_{2 n+1, l}$ be an element such that for all matrices

$$
\left(\begin{array}{ccc}
P & V & Q \\
\Phi & U & \Psi \\
R & W & S
\end{array}\right) \in H_{2 n+1, l} \cdot M
$$

it holds that $\operatorname{rk}(R) \leq m$ or the first and last column of $W$ are linearly dependent. Then we have $\operatorname{rk}(M) \leq c_{1} m+c_{0}$.

Proof. Let

$$
\left(\begin{array}{lll}
P & V & Q \\
\Phi & U & \Psi \\
R & W & S
\end{array}\right)
$$

be an element of the orbit of $M$. We assume that $c_{2} m \leq n$ with $c_{2}$ high enough and we will prove a series of claims, which together imply that $\operatorname{rk}(M) \leq c_{1} m+c_{0}$ for suitable $c_{0}, c_{1} \in \mathbb{N}$.

(x) We have $\operatorname{rk}(R) \leq m+4$.

Suppose that $\operatorname{rk}(R)>m$. Note that $\operatorname{Diag}\left(I_{l n}, g, I_{l n}\right) \in H_{2 n+1, l}$ for all $g \in \mathrm{GL}_{l}$ with $g J g^{T}=J$. We have

$$
\left(\begin{array}{lll}
I_{l n} & & \\
& g & \\
& & I_{l n}
\end{array}\right)\left(\begin{array}{ccc}
P & V & Q \\
\Phi & U & \Psi \\
R & W & S
\end{array}\right)\left(\begin{array}{lll}
I_{l n} & & \\
& g & \\
& & I_{l n}
\end{array}\right)^{-1}=\left(\begin{array}{ccc}
P & V g^{-1} & Q \\
g \Phi & g U g^{-1} & g \Psi \\
R & W g^{-1} & S
\end{array}\right)
$$

for all $g \in \mathrm{GL}_{l}$. So we see that the first and last column of $\mathrm{Wg}^{-1}$ are linearly dependent for all $g \in \mathrm{GL}_{l}$ with $g J g^{T}=J$. Using the fact that

$$
g=\left(\begin{array}{ccccc}
1 & & & & \\
& \ddots & & & \\
\lambda & & \ddots & & \\
& & & \ddots & \\
& & -\lambda & & 1
\end{array}\right)
$$

satisfies $g J g^{T}=J$ as long as $\lambda$ is not in the middle row together with $J J J^{T}=J$, it is now easy to check that $\operatorname{rk}(W) \leq 2$. Next, note that

$$
\left(\begin{array}{ccc}
I_{l n} & A & -\frac{1}{2} A J A^{T} \\
& I_{l} & -J A^{T} \\
& & I_{l n}
\end{array}\right) \in H_{2 n+1, l}
$$

for all $A \in K^{l n \times l}$. For all $A \in K^{\ln \times l}$, we have

$$
\left(\begin{array}{ccc}
I_{l n} & A & -\frac{1}{2} A J A^{T} \\
& I_{l} & -J A^{T} \\
& & I_{l n}
\end{array}\right)^{-1}\left(\begin{array}{ccc}
P & V & Q \\
\Phi & U & \Psi \\
R & W & S
\end{array}\right)\left(\begin{array}{ccc}
I_{l n} & A & -\frac{1}{2} A J A^{T} \\
& I_{l} & -J A^{T} \\
& & I_{l n}
\end{array}\right)=\left(\begin{array}{ccc}
\bullet & \bullet & \bullet \\
\bullet & \bullet & \bullet \\
R & W+R A & \bullet
\end{array}\right)
$$

and hence $\operatorname{rk}(W+R A) \leq 2$. So $\operatorname{rk}(R A) \leq 4$ and hence $\operatorname{rk}(R) \leq 4$.

(y) We have $\operatorname{rk}(Q) \leq m+4$ and $\operatorname{rk}(P)=\operatorname{rk}(S) \leq 3(m+4) / 2$. 
Repeat the proof of Lemma 46 and act with matrices

$$
\left(\begin{array}{ccc} 
& & I_{l n} \\
& I_{l} & \\
I_{l n} & & A
\end{array}\right),\left(\begin{array}{lll}
I_{l n} & & B \\
& I_{l} & \\
& & I_{l n}
\end{array}\right)
$$

with $A=-A^{T}$ and $B=-B^{T}$.

(z) We have $\operatorname{rk}(W)=\operatorname{rk}(\Phi), \operatorname{rk}(V)=\operatorname{rk}(\Psi) \leq 4(m+4)$ and $\operatorname{rk}(U) \leq 22(m+4)$.

We have

$$
\left(\begin{array}{ccc}
I_{l n} & A & -\frac{1}{2} A J A^{T} \\
& I_{l} & -J A^{T} \\
& & I_{l n}
\end{array}\right)^{-1}\left(\begin{array}{ccc}
P & V & Q \\
\Phi & U & \Psi \\
R & W & S
\end{array}\right)\left(\begin{array}{ccc}
I_{l n} & A & -\frac{1}{2} A J A^{T} \\
& I_{l} & -J A^{T} \\
& & I_{l n}
\end{array}\right)=\left(\begin{array}{ccc}
\bullet & \bullet & \bullet \\
\bullet & \bullet & \bullet \\
\bullet & \bullet & T
\end{array}\right)
$$

with $T=-\frac{1}{2} R A J A^{T}-W J A^{T}+S$ for all $A \in K^{\ln \times l}$. So $\operatorname{rk}\left(W J A^{T}\right) \leq 4(m+4)$ for all $A \in K^{l n \times l}$. So $\operatorname{rk}(W)=\operatorname{rk}(\Phi) \leq 4(m+4)$. By conjugating with

$$
\left(\begin{array}{ccc} 
& & I_{l n} \\
& I_{l} & \\
I_{l n} & &
\end{array}\right)
$$

we also see that $\operatorname{rk}(V)=\operatorname{rk}(\Psi) \leq 4(m+4)$. We have

$$
\left(\begin{array}{ccc}
I_{l n} & A & -\frac{1}{2} A J A^{T} \\
& I_{l} & -J A^{T} \\
& & I_{l n}
\end{array}\right)^{-1}\left(\begin{array}{ccc}
P & V & Q \\
\Phi & U & \Psi \\
R & W & S
\end{array}\right)\left(\begin{array}{ccc}
I_{l n} & A & -\frac{1}{2} A J A^{T} \\
& I_{l} & -J A^{T} \\
& & I_{l n}
\end{array}\right)=\left(\begin{array}{lll}
\bullet & \bullet & T \\
\bullet & \bullet & \bullet \\
\bullet & \bullet & \bullet
\end{array}\right)
$$

with

$$
T=\left(\begin{array}{lll}
I_{l n} & -A & -\frac{1}{2} A J A^{T}
\end{array}\right)\left(\begin{array}{lll}
P & V & Q \\
\Phi & U & \Psi \\
R & W & S
\end{array}\right)\left(\begin{array}{c}
-\frac{1}{2} A J A^{T} \\
-J A^{T} \\
I_{l n}
\end{array}\right) .
$$

Now, we know that $\operatorname{rk}(T) \leq m+4$. Also, the matrix $T$ is a sum of nine matrices: the matrix $A U J A^{T}$ and eight other matrices for which we have found bounds on the rank. Adding all these bounds together, we find that

$$
\operatorname{rk}\left(A U J A^{T}\right) \leq(1+1+1+3 / 2+3 / 2+4+4+4+4)(m+4)=22(m+4)
$$

for all $A \in K^{l n \times l}$. Hence $\operatorname{rk}(U) \leq 22(m+4)$.

Together (x), (y) and (z) show that

$$
\operatorname{rk}\left(\begin{array}{ccc}
P & V & Q \\
\Phi & U & \Psi \\
R & W & S
\end{array}\right) \leq c_{1} m+c_{0}
$$

for some $c_{0}, c_{1} \in \mathbb{N}$. So this holds in particular if we let this matrix be $M$ itself.

We combine these results as in the previous section. Lemmas 49 and 50 play the roles of Lemmas 44 and 45 and give us off-diagonal polynomials. Then, Proposition 51 with $k=2$ shows us the structure of the off-diagonal part of the matrix as a $\mathrm{GL}_{n}$-representation with the Zariski topology. From this and the degree of the off-diagonal polynomial, we get bounds on ranks of some submatrices. Lemma 52 turns these bounds into a rank bound on the matrix itself. Finally, we find similarly to Lemma 30 that $X \subseteq\{0\}$ and this implies that $V$ is G-Noetherian. 


\section{Further Questions}

Representation-inducing functors. As stated in the introduction, many examples of infinite-dimensional spaces that are Noetherian up to the action of some group arise from taking limits of sequences after applying certain functors. So one could hope that our spaces $V$ and groups $G$ can be contructed from functors in such a way that these functors are suitably Noetherian and that this Noetherianity implies the results of this paper. Concretely, is there a class of topologically Noetherian functors from which the representations in this paper arise and do any new representations arise from such functors?

Classifications for types B, C and D. Theorem 13 classifies all G-stable closed subsets of $V$ when $G$ is the direct limit of diagonal embeddings between classical groups of type A. One wonders whether such a classification exists for the other types. The key part of the proof of Theorem 13 seems to be Proposition 22 . which gives a complete descriptions of the closures of orbits. So it would be very interesting to see whether such descriptions can be found for the other types.

\section{REFERENCES}

[ADF] S. Abeasis, A. Del Fra, Young diagrams and ideals of Pfaffians, Adv. Math. 35 (1980), no. 2, pp. 158-178.

[BZ] A.A. Baranov, A.G. Zhilinskii, Diagonal direct limits of simple Lie algebras, Comm. Algebra 27 (1999), no. 6, pp. 2749-2766.

[BA] H.L. Bodlaender, B. van Antwerpen-de Fluiter, Reduction Algorithms for Graphs of Small Treewidth, Inf. Comput. 167 (2001), no. 2, pp. 86-119.

[Dr] J. Draisma, Topological Noetherianity of polynomial functors, J. Am. Math. Soc. 32 (2019), no. 3, pp. 691-707.

[DE] J. Draisma, R.H. Eggermont, Plücker varieties and higher secants of Sato's Grassmannian, J. Reine Angew. Math. 737 (2018), pp. 189-215.

[Eg] R.H. Eggermont, Finiteness properties of congruence classes of infinite matrices, Linear Algebra Appl. 484 (2015), pp. 290-303.

[ES] R.H. Eggermont, A. Snowden, Topological noetherianity for algebraic representations of infinite rank classical groups, preprint.

[HS] C.J. Hillar, S. Sullivant, Finite Gröbner bases in infinite dimensional polynomial rings and applications, Adv. Math. 229 (2012), no. 1, pp. 1-25.

[St] A. Stasinski, Similarity and commutators of matrices over principal ideal rings, Trans. Amer. Math. Soc 368 (2016), no. 4, pp. 2333-2354.

[SS] B. Sturmfels, S. Sullivant, Combinatorial secant varieties, Pure Appl. Math. Q. 2 (2006), no. 3, pp. 867-891.

[Ta] O. Taussky, The role of symmetric matrices in the study of general matrices, Linear Algebra Appl. 5 (1972), no. 2, pp. 147-154.

Universität Bern, Mathematisches Institut, Alpeneggstrasse 22, 3012 Bern, Switzerland

E-mail address: arthur.bik@math.unibe.ch 